\title{
Stochastic neural network model for spontaneous bursting in hippocampal slices
}

\author{
B. Biswal ${ }^{1,2}$ and C. Dasgupta ${ }^{3,4}$ \\ ${ }^{1}$ ICA1, University of Stuttgart, Pfaffenwaldring 27, D-70569 Stuttgart, Germany \\ ${ }^{2}$ Department of Physics, Sri Venkateswara College, University of Delhi, New Delhi, India \\ ${ }^{3}$ Department of Physics, Indian Institute of Science, Bangalore 560 012, India \\ ${ }^{4}$ Condensed Matter Theory Unit, JNCASR, Bangalore 560 064, India
}

\begin{abstract}
A biologically plausible, stochastic, neural network model that exhibits spontaneous transitions between a low-activity (normal) state and a high-activity (epileptic) state is studied by computer simulation. Brief excursions of the network to the high-activity state lead to spontaneous population bursting similar to the behavior observed in hippocampal slices bathed in a high-potassium medium. Although the variability of interburst intervals in this model is due to stochasticity, first return maps of successive interburst intervals show trajectories that resemble the behavior expected near unstable periodic orbits (UPOs) of systems exhibiting deterministic chaos. Simulations of the effects of the application of chaos control, periodic pacing, and anticontrol to the network model yield results that are qualitatively similar to those obtained in experiments on hippocampal slices. Estimation of the statistical significance of UPOs through surrogate data analysis also leads to results that resemble those of similar analysis of data obtained from slice experiments and human epileptic activity. These results suggest that spontaneous population bursting in hippocampal slices may be a manifestation of stochastic bistable dynamics, rather than of deterministic chaos. Our results also question the reliability of some of the recently proposed, UPO-based, statistical methods for detecting determinism and chaos in experimental time-series data.
\end{abstract}

\section{INTRODUCTION}

With the development of many new techniques of nonlinear time-series analysis [1], a number of recent studies have attempted to understand brain dynamics [2] in the context of deterministic chaos [3]. Experimental studies on normal brain activity [4] and epilepsy [5] have looked for signature of chaos. New hypotheses $[6,7]$ have been proposed for possible roles of chaos in the functioning of the brain. A convincing demonstration of the occurrence of deterministic chaos in brain dynamics is of fundamental importance in understanding the collective behavior of neuronal networks in the brain. It may also lead to the possibility of short-term prediction and control, with potentially important medical applications.

Due to the large number of neurons involved in any specific brain function, the underlying dynamics is inherently high dimensional. This makes reliable detection of lowdimensional chaos in brain signals a formidable task. Datasets from physiological systems are often inadequately small, nonstationary, and contaminated with noise, posing severe problems for a statistically reliable time-series analysis. Though a number of recent studies have reported the detection of low-dimensional chaos through time-series analysis of brain signals, experts often remain skeptical about such claims [8].

The results of an in vitro experiment of Schiff et al. [9] on spontaneous population bursting in rat hippocampal slices bathed in a medium with high potassium concentration have attracted much attention in this context. In this work, the presence of unstable periodic orbits (UPOs) in the dynamics of the neuronal network is inferred from an analysis of the first return map of successive interburst intervals (IBIs).
Since UPOs are known to be embedded in typical chaotic attractors, the presence of UPO-like trajectories suggests the occurrence of deterministic chaos. Then a variant of a chaos control technique proposed by Ott, Grebogi and Yorke [10] is used to attempt to make the IBIs more uniform. Application of this technique is found to make the bursts more periodic. These observations are interpreted as evidence for deterministic chaos in the neuronal population bursting behavior. Results of periodic pacing and the application of an "anticontrol" method designed to make the IBIs more irregular are also reported. Since the spontaneous bursting observed in this in vitro experiment exhibits many similarities with interictal spikes in human epilepsy, it is suggested that human epileptic activity may also be chaotic and therefore amenable to methods of chaos control and anticontrol. The possibility of application of these methods in the treatment of human epilepsy has attracted wide attention [11].

Some of the methodology and conclusions of the brainslice experiment have been questioned by Christini and Collins [12] who have shown that the method of chaos control used by Schiff et al. works equally well in some simple stochastic systems. These authors consider the effects of additive noise on the behavior of the FitzHugh-Nagumo equations which describe a single spiking neuron. The presence of the noise causes the interspike interval (ISI) to be irregular. The first return map of successive ISIs shows evidence for the existence of (apparent) unstable fixed points, and the application of the chaos control method makes the ISIs more regular. The qualitative behavior found in this simple system is similar to that found in the experiment of Schiff et al. This work casts doubt on the interpretation of the results of the brain-slice experiment as evidence for the occurrence of deterministic chaos. 
More recently, several surrogate methods $[13,14]$ to assess the statistical significance of UPOs detected from time-series analysis have been proposed. Applications of some of these methods to the time-series data of the brain-slice experiment claim $[15,16]$ to have reestablished the original conclusion of Schiff et al. on the existence of deterministic chaos in the bursting dynamics of hippocampal slices. Results similar to those in Refs. [9] and [16] have been reported [17,18] recently for hippocampal slices studied under three different experimental conditions [high potassium, zero magnesium, and in the presence of $g$-amino butyric acid $\left(\mathrm{GABA}_{A}\right)$ receptor antagonists]. A similar conclusion about the occurrence of chaos in epileptic brain activity has also been reached from a surrogate analysis [19] of time-series data recorded from a human subject.

In this paper, we address the issue of whether or not the spontaneous population bursts found in brain-slice preparations and analogous interictal spikes in the human epileptic brain are manifestations of deterministic chaos. Our work involves extensive simulations of an existing neural network model for focal epilepsy [20]. This network exhibits a lowactivity (normal) attractor and a high-activity (epileptic) one. Brief excursions of the network to the high-activity state in the absence of any external stimulus appear as spontaneous bursts analogous to those observed in the brain-slice experiment. In our model, however, the irregularity of the IBIs is strictly a consequence of stochasticity arising from the randomness of the sequence in which the neurons are updated. The network model and its bursting dynamics are described in Sec. II.

Although the variability of the IBIs in our network model is a consequence of stochasticity, we find trajectories that may be interpreted as evidence for the presence of (apparent) UPOs [unstable fixed points (UFPs) in the first return map]. This allows us to apply the methods of chaos control, periodic pacing, and anticontrol considered by Schiff $e t$ al. to our stochastic neural network model. We have also studied the effects of demand pacing on the network dynamics. Results of our simulations of the effects of these control methods are described in detail in Sec. III. These results are found to be quite similar to those obtained in brain-slice experiments.

Section IV contains the results of surrogate analysis for the statistical significance of the UPO-like trajectories found in the IBI datasets. Three different methods are considered in our analysis. These methods include the ones used recently to claim evidence for the presence of statistically significant UPOs in time-series data obtained from brain-slice experiments $[16,17]$ and from human epileptic activity [19]. Applications of these methods to the IBI time series obtained from simulations of our stochastic neural network model yield results that are similar to some of those reported in Refs. $[16,17,19]$.

Thus, our stochastic neural network model with bistable dynamics reproduces most of the results obtained in brainslice experiments. This result casts doubt on the interpretation of the experimental data as evidence for deterministic chaos, and suggests that epileptic brain activity may be a manifestation of stochastic bistability. Our work also questions the reliability of some of the UPO-based statistical methods used in recent studies $[16,17,19]$ to infer the presence of determinism and chaos in experimental time-series data. A summary of our main results and conclusions is presented in Sec. V. Some of the results reported here were summarized in a recent Letter [21].

\section{THE NEURAL NETWORK MODEL AND ITS DYNAMICS}

In this section, we define the neural network model used in our simulations and describe its bursting dynamics. This model was first proposed by Mehta, Dasgupta, and Ullal [20] for describing the process of "kindling" [22] of focal epilepsy. Kindling is a phenomenon in which a part of the brain is externally stimulated, either through electric pulses or through chemicals, to produce spontaneous epileptic activity. It is believed that kindling provides a good animal model for the development of an understanding of epilepsy in the mammalian brain. In the present work, we have used kindled networks to model spontaneous population bursting in hippocampal slices and interictal spikes in human epilepsy.

\section{A. The model}

The network consists of $N$ McCulloch-Pitts (binary) neurons $\left\{S_{i}\right\}, i=1,2, \ldots, N$, each of which is excitatory and can take the two values 0 and 1, representing low and high firing rates, respectively. The inhibitory neurons are collectively modeled by a background inhibition, which is assumed to be proportional to the number of active excitatory neurons. Our model with binary neurons is clearly inappropriate for describing phenomena that depend crucially on the detailed biophysical properties of individual neurons. However, such models are expected [23] to be adequate for qualitative descriptions of the collective dynamical behavior of biological networks.

The "local field" $h_{i}(t)$ at the $i$ th neuron at time $t$ is given by

$$
\begin{aligned}
h_{i}(t)= & \sum_{j=1}^{N}\left[\left\{J_{i j} S_{j}(t)-w S_{j}(t)\right\}\right. \\
& \left.+\lambda\left\{K_{i j} S_{j}(t-\tau)-w S_{j}(t-\tau)\right\}\right],
\end{aligned}
$$

where $w$ is the relative strength of inhibition, $\lambda$ is the relative strength of the delayed signal, and $\tau$ is the time delay associated with the delayed signal. The four terms from left in Eq. (1) represent, respectively, fast global excitation, fast global inhibition, slow global excitation, and slow global inhibition. These four ingredients are believed [24] to be essential for a realistic modeling of neural oscillations in the hippocampus. The "time" $t$ is discrete, with each unit (referred to as one "pass") corresponding to the updating of $N$ neurons. The neurons are updated one at a time in a random sequence, according to the rule $S_{i}(t+1)=1$ if $h_{i}(t) \geqslant 0$ and $S_{i}(t+1)=0$ if $h_{i}(t)<0$.

A fixed number $(q)$ of random low-activity patterns (memories) $\left\{\xi_{i}^{\mu}\right\}, i=1,2, \ldots, N ; \mu=1,2, \ldots, q$, are stored in the synaptic matrices $J_{i j}$ and $K_{i j}$ in the following way: 


$$
\begin{aligned}
J_{i j} & =1 \quad \text { if } \quad \sum_{\mu=1}^{q} \xi_{i}^{\mu} \xi_{j}^{\mu}>0, \\
& =0 \quad \text { otherwise, }
\end{aligned}
$$

and

$$
\begin{aligned}
K_{i j} & =1 \quad \text { if } \sum_{\mu=1}^{q} \xi_{i}^{\mu+1} \xi_{j}^{\mu}>0, \\
& =0 \quad \text { otherwise, }
\end{aligned}
$$

with $\xi_{i}^{q+1}=\xi_{i}^{1}$, and $J_{i i}=K_{i i}=0$. To simulate low average activity of the network in the absence of any external stimulus, the net activity of each memory, $\sum_{i=1}^{N} \xi_{i}^{\mu}$, is set at a value $n \ll N$. The $n$ "active" neurons in each memory are chosen randomly. The "fast" synaptic connections $J_{i j}$ act to stabilize the system in one of the memory states, while the "slow" connections $K_{i j}$ tend to induce transitions from a memory state to the next one in the sequence after $\sim \tau$ passes. The global inhibition represented by the $w$ terms prevents simultaneous activation of more than one memories. With appropriate choice of the values of the parameters (specifically, for $\lambda>1$ and $w<1$ ), the network exhibits a lowactivity limit cycle in which all the $q$ memories are visited sequentially [20].

The simulations carried out in this study are for the following parameter values: $N=200, q=20, n=10, w=0.6$, $\lambda=2, \tau=2$ passes. Proper functioning of the network in the absence of any external input (the resting state) is verified by starting the network in the memory state $\xi_{i}^{1}$ at time $t=0$, and then monitoring the time evolution of the overlaps, $m^{\mu}(t)$ $=\sum_{i=1}^{N} \xi_{i}^{\mu} S_{i}(t), \mu=1,2, \ldots, q$, of the network with the memory states. The network evolution is found to follow a limit cycle in which it traverses sequentially the 20 memories in about 60 passes. The resulting low-amplitude, noisy oscillations of the net activity, $S_{u p}(t)=\sum_{i=1}^{N} S_{i}(t)$, are shown in Fig. 1(a).

\section{B. Spontaneous population bursting}

To simulate the excess excitability of brain slices bathed in a high-potassium medium that induces spontaneous bursting, the network is "chemically kindled" [20] through a Hebbian learning mechanism defined in the following way. If, over $n_{1}$ consecutive passes, $S_{i}=1$ and $S_{j}=1$ more often than $n_{2}$ times, then the fast synaptic strength $J_{i j}$ between the two neurons is permanently set to 1 . In our simulation, $n_{1}$ $=10$ and $n_{2}=6$. The networks are chemically kindled by switching on the Hebbian learning for 50 initial passes under reduced inhibition ( $w$ is reduced from 0.6 to 0.24 during this period). The Hebbian learning process generates many new excitatory synaptic connections, leading to increased connectivity between different memories. This, in turn, leads to the formation of a new high-activity (epileptic) attractor of the dynamics corresponding to excess correlated firing of the neurons. Different networks (i.e., networks with different re-
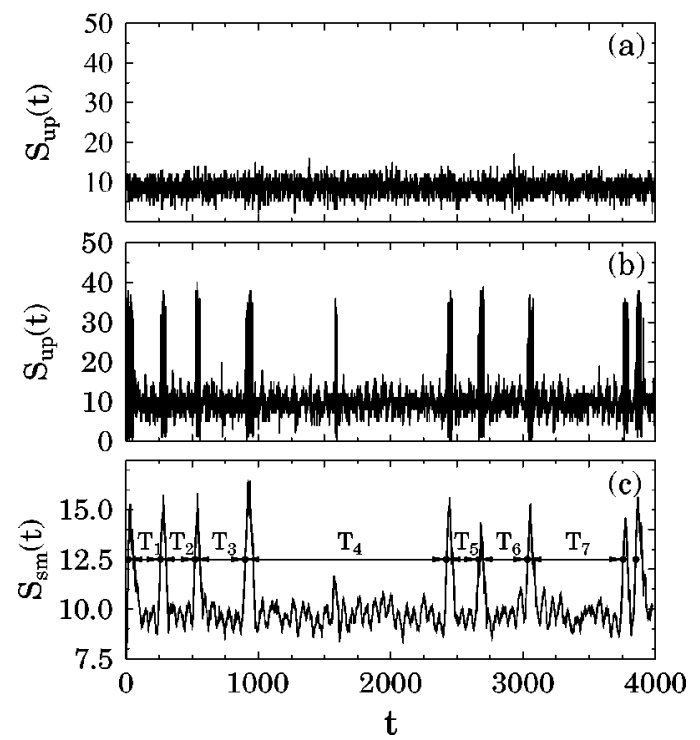

FIG. 1. (a) Network dynamics before kindling, showing noisy, low-amplitude oscillations of the net activity $S_{u p}$ around an average value near $n=10$; (b) dynamics of the kindled network, showing spontaneous bursting at irregular time intervals; (c) smoothened network activity with IBIs $\left(T_{1}, T_{2}\right.$ etc.) computed from positive threshold crossings denoted by dark circles. These results were obtained using random sequential updating.

alizations of the random memories $\left\{\xi_{i}^{\mu}\right\}$ ) exhibit varying degrees of kindling due to differences in the initial connectivity pattern.

For random sequential updating of the neurons, the kindled network makes occasional, short-lived, spontaneous transitions to the high-activity attractor [20]. This is similar to the spontaneous population bursting observed in the brainslice experiment. These bursts of high correlated activity appear as "spikes" in the net activity of the network. The IBIs are measured using the threshold crossing method [12]. Like a low-pass filter in experiments, the net activity, $S_{u p}(t)$, is first smoothened to $S_{s m}(t)=1 / t_{s m} \Sigma_{t^{\prime}=t-t_{s m}+1}^{t} S_{u p}\left(t^{\prime}\right)$, with $t_{s m}=40$ passes. Whenever $S_{s m}(t)$ crosses an appropriately chosen upper threshold in the positive direction, a burst is recorded at the corresponding time $t$. Before the occurrence of the next burst, $S_{s m}(t)$ must decrease below a lower threshold corresponding to the low-activity state of the network. To avoid rare occurrences of persistent high activity, refractoriness is introduced in the network in the following manner. If $S_{s m}(t)$ remains higher than the upper threshold value continuously for 20 passes, the neuron configurations (the current one as well as the previous ones accounting for the delayed signal) are reset to one of the low-activity memory patterns chosen randomly.

The net activity of a kindled network with refractoriness is shown in Fig. 1(b), and the smoothened net activity is plotted in Fig. 1(c). Positive crossings denoted by dark circles correspond to the occurrence of bursts and $T_{1}, T_{2}, \ldots$, are the IBIs. Individual IBIs are integers measured in units of passes.

We have studied 10 networks with different realizations of the random memories. A detailed analysis was carried out for 


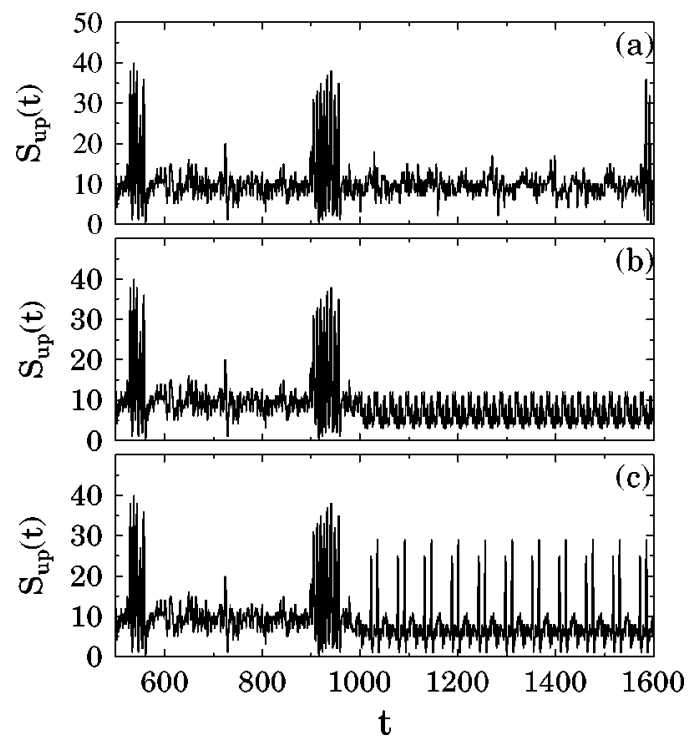

FIG. 2. (a) Network dynamics under random sequential updating, showing excursions to the high-activity attractor at irregular time intervals, and noisy low-amplitude oscillations of the net activity $S_{u p}(t)$ between these excursions; (b) fixed sequence updating (switched on at $t=1000$ ) that traps the network in the low-activity attractor, leading to low-amplitude periodic behavior of $S_{u p}(t)$; (c) fixed sequence updating (switched on at $t=1000$ ) that produces periodic transitions to the high-activity attractor.

six of these networks. Each network shows a wide distribution of IBIs and the value of the average IBI varies substantially from one network to another, reflecting different degrees of kindling. Similar sample-to-sample variations have been found [25] in hippocampal slices very similar to those used in the experiment of Ref. [9]. The variability of IBIs shown in the raw-data plots in Ref. [25] is qualitatively similar to that found in our simulations.

\section{Stochasticity of the bursting dynamics}

The irregular bursting shown in Fig. 1(b) is found only for random sequential updating (RSU), i.e., when the neurons are updated one at a time in a random sequence. Operationally, one of the $N$ neurons is selected randomly for updating, its local field is calculated, and its state variable $S_{i}$ is set to 0 or 1 depending on the sign of the local field. This process is repeated $N$ times to complete one pass. To ascertain the nature of the dynamics, we also checked the time evolution of the network without the randomness in the updating scheme and the refractoriness. In these simulations, refractoriness was implemented by always resetting the network to a fixed memory and the network was updated following two different deterministic schemes: fixed sequential updating (FSU) and parallel updating (PU).

In the FSU, a specific sequence for updating the $N$ neurons is chosen randomly, and then the neurons are always updated in that sequence. For most choices of the update sequence, the network remains confined to the low-activity attractor and $S_{u p}$ exhibits perfectly periodic, low-amplitude oscillations. This is shown in Fig. 2(b). However, for certain "special" choices of the update sequence, the network is found to make periodic transitions to the high-activity attractor, as shown in Fig. 2(c). In the PU scheme, all the neurons are updated simultaneously. The dynamics under PU is qualitatively similar to the behavior found for FSU. Depending on the network state at the time of switching on the PU, the network either remains confined to the low-activity attractor or makes periodic transitions to the high-activity attractor with somewhat higher degree of correlated activity than that found for FSU. These observations establish that the underlying stochasticity in the updating scheme is an essential requirement for the network to exhibit spontaneous bursting similar to that observed in brain slices (large variability in the IBIs).

Among the three different updating procedures discussed above, the RSU scheme also appears to be most "physical" for describing the behavior of brain slices. A realistic description of the dynamics of a network of biological neurons may be obtained [26] in terms of coupled differential equations that govern the time evolution of membrane potentials and ionic currents. In this description, a neuron fires (i.e., generates an action potential) if its net membrane potential exceeds a threshold. Once a neuron fires, it cannot fire again unless a short refractory period has elapsed. In the discretetime dynamics of our simple model, the unit of time (one pass) approximately corresponds to this refractory period [23]: each neuron can, on an average, fire once during this period. However, if no external "clocklike" mechanism is present, then there is no reason to expect that the membrane potentials of different neurons in a network would reach the threshold value at exactly the same time. Since external inputs that may synchronize or order in time the firing of all the neurons are not expected to be present in such "in vitro" brain slices, the asynchronous dynamics of the RSU scheme should be appropriate for describing such systems. We, therefore, used the RSU scheme in all our simulations. The results described above suggest that the time series of IBIs obtained from these simulations are stochastic.

We have carried out several tests to check whether or not the IBI time series are truly stochastic. We looked for correlations in the IBI time series by calculating the autocorrelation function

$$
C(n)=\frac{1}{m-n} \sum_{i=1}^{m-n}\left(T_{i}-\bar{T}\right)\left(T_{i+n}-\bar{T}\right) / \sigma^{2},
$$

where $m$ is the total number of IBIs in the dataset, $\bar{T}$ $=1 / m \sum_{i=1}^{m} T_{i}$ is the average IBI, and $\sigma^{2}=1 / m \sum_{i=1}^{m}\left(T_{i}\right.$ $-\bar{T})^{2}$. As shown in Fig. 3, the IBIs are completely uncorrelated in time in all the networks. A similar absence of correlation has been found in the experiment of Ref. [25] on hippocampal slices.

The probability distribution of the IBIs follows a Poisson form, $P\left(T_{n}\right) \propto \exp \left(-\alpha T_{n}\right)$, in all the networks. Sample results for network 1 are presented in Fig. 4, in which both the simulation data and the fit to a Poisson form are shown. The smoothening procedure we have used for low-pass filtering of the data does not allow the occurrence of IBIs smaller than about $2 t_{s m}=80$ passes. For this reason, the Poisson dis- 


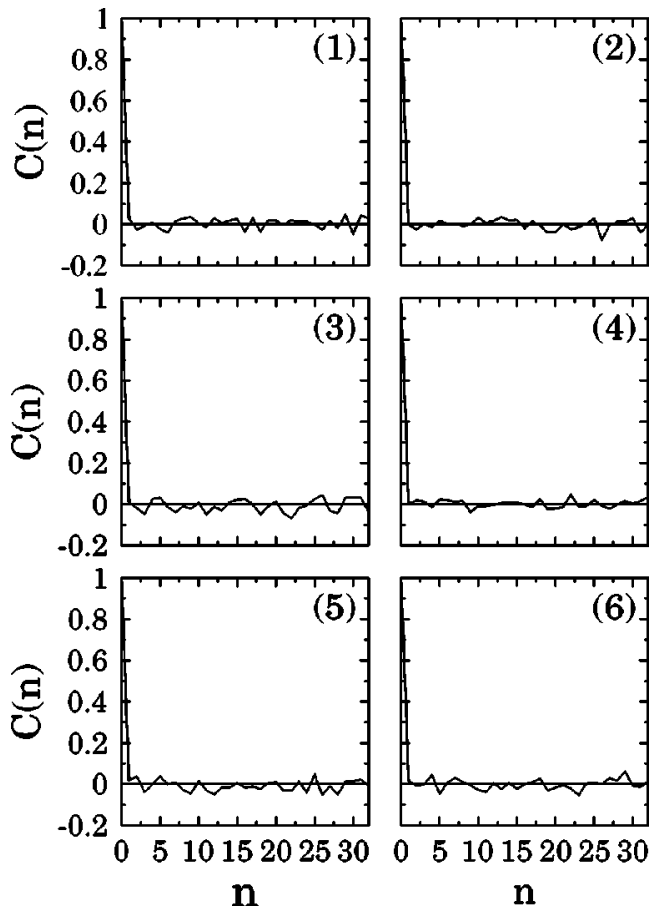

FIG. 3. Autocorrelation function $C(n)$ of the IBIs from six networks.

tribution is cut off near the origin. The value of the parameter $\alpha$ shows large sample-to-sample variation in the range between $8 \times 10^{-4}$ and $3 \times 10^{-3}$. The form of the distribution of the IBIs recorded in brain-slice experiments $[9,17,25]$ has not been reported in the literature. The data in the first return maps of the IBI time series shown in Refs. $[17,18]$ indicate a distribution that exhibits a sharp maximum close to the low end and decreases slowly to zero at the high end. This is similar to a Poisson distribution truncated at the low end.

Our results suggest that the transition of the network from the low-activity state to the high-activity one is a stochastic process that has a small probability $p=1-\exp (-\alpha)$ of occurring at each step. For this value of $p$, the probability of having two successive transitions separated by a time interval of $T_{n}$ units would be proportional to $(1-p)^{T_{n}}$

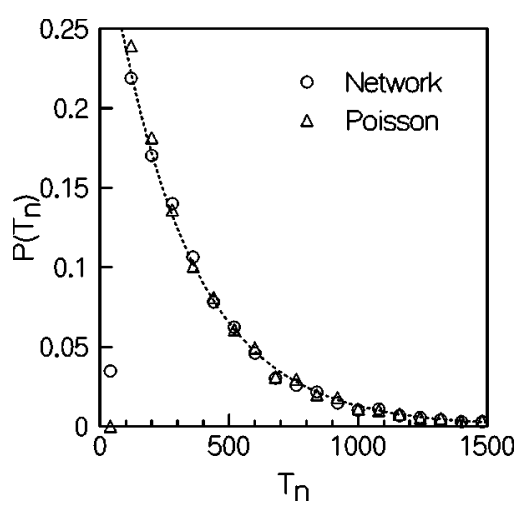

FIG. 4. Probability distribution of IBIs from network simulation (circles) and Poisson simulation (triangles) in the absence of control in network 1. The dashed line is the best fit to an exponential (Poisson) form. $=\exp \left(-\alpha T_{n}\right)$, which would match the Poisson distribution of the IBIs found in our simulations. To check this, we have carried out Monte Carlo simulations of a Poisson process with probability $p$ of occurrence at each time step. In this simulation, a random number $r$ distributed uniformly between 0 and 1 is generated at each time step, and the occurrence of a "burst" is recorded if $r \leqslant p$. Since $r$ is distributed uniformly between 0 and 1 , the probability of $r$ being less than or equal to $p$ is $p$ itself. So, this Monte Carlo procedure ensures that the bursts occur with probability $p$. Once a burst occurs, the next one cannot occur during the next $2 t_{s m}$ time steps. We use this procedure to generate the same number of "IBIs" as in the original time series and compute their distribution function. As shown in Fig. 4, the distribution functions obtained from the network and Poisson simulations are essentially identical. Similar close agreements between the results of the network and Poisson simulations of the behavior under different control procedures (see Sec. III F for details) provide strong support to the conclusion that the IBI time series obtained in our simulations are stochastic in nature.

A defining feature of deterministic chaos is extremely sensitive dependence on initial conditions, manifested in an exponential divergence of trajectories starting from nearby points. We have carried out a simple calculation to look for this behavior in our IBI time series. This involves a calculation of the quantity $\langle\ln [d(k)]\rangle$ where $d(k)=\left|T_{i+k}-T_{j+k}\right|$, and the average $\langle\cdots\rangle$ is over pairs $i$ and $j$ with $d(0)=\mid T_{i}$ $-T_{j} \mid<n, n$ being a small number. A similar calculation for the chaotic logistic map shows an exponential growth of $\langle d(k)\rangle$ with increasing $k$, and a crude estimate of the value of the Lyapunov exponent can be obtained by fitting the dependence of $\langle\ln [d(k)]\rangle$ on $k$ to a straight line. For our IBI time series, on the other hand, we find that $\langle\ln [d(k)]\rangle$ is independent of both $k$ and $n$, indicating that the IBIs are independent random variables. Similar results are obtained for a twodimensional delay embedding,

$$
\begin{gathered}
d(0)=\left|T_{i}-T_{j}\right|+\left|T_{i+1}-T_{j+1}\right|<n, \\
d(k)=\left|T_{i+k}-T_{j+k}\right|+\left|T_{i+k+1}-T_{j+k+1}\right| .
\end{gathered}
$$

Here, we find that $\langle\ln [d(k)]\rangle$ is independent of $n$, and independent of $k$ for all $k>1$. These observations confirm the stochastic nature of the $T_{i}$ time series and indicate that the use of more sophisticated methods to calculate Lyapunov exponents from our time-series data would not be meaningful. Further evidence for the stochastic nature of the IBI time series is obtained from the surrogate analysis described in Sec. IV.

The behavior of $d(k)$ found for our IBI time series is similar to the observation in Ref. [17] that a small cluster of points in the first return map of the experimentally obtained IBI time series expands rapidly and covers nearly the whole allowed region within two iterations. Due to this rapid expansion, conventional methods of estimating the value of the largest Lyapunov exponent from time-series data could not be used to analyze the experimental data. Instead, a new technique called "expansion rate analysis" [17] was used. 


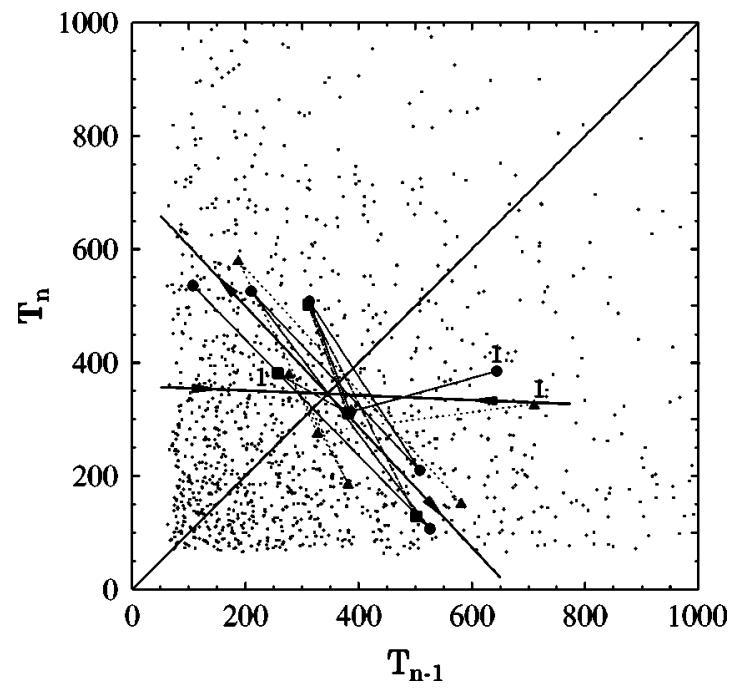

FIG. 5. Recurrent UPO-like trajectories in network 1 chosen for control. Three sequences starting at different times (denoted by 1) are shown along with the calculated stable and unstable manifolds denoted by arrows.

This analysis did not provide any evidence of determinism in data. This feature of the experimentally obtained IBI time series is clearly in agreement with our simulation results.

\section{SIMULATIONS OF CONTROL METHODS}

As discussed in the preceding section, the IBI time series obtained from simulations of the stochastic neural network exhibit general characteristics that are quite similar to those of the IBI time series obtained in brain-slice experiments $[9,25]$. It is, therefore, interesting to enquire whether some of the control results reported in Ref. [9] can also be reproduced in simulations of the network model. We have carried out extensive simulations of the behavior of six networks under the application of various control methods. The results of these simulations are described in this section.

\section{A. Apparent unstable periodic orbits}

Following the analysis of IBIs from spontaneously bursting brain slices [9], we searched for UPO-like trajectories in first return maps of IBIs from six different networks. UPOs of period one appear as UFPs in the first return map defined as a plot of $T_{n}$ vs $T_{n-1}$. Unlike typical return maps from deterministic chaotic dynamics, the points in the return maps constructed from our IBI time series fill up the entire space, reflecting the underlying stochastic dynamics of the network. The Poisson distribution of the IBIs leads to a clustering of points near the origin and the axes. A return map constructed from a 1320 -IBI time series collected over $5 \times 10^{5}$ passes for network 1 is shown in Fig. 5. The lack of any geometrical structure in the return maps of our IBI time series is similar to the behavior found in brain-slice experiments $[9,17,18]$.

Although the return maps do not have the structure expected for deterministic dynamics, we found many UPO-like trajectories which have multiple occurrences and satisfy all the criteria adopted by Schiff et al. [9]. Many such purely accidental occurrences of UPO-like trajectories were found in all the six networks studied. The apparent UPOs are identified from trajectories in the return map that first approach a point on the identity line $\left(T_{n}=T_{n-1}\right)$ along a line (stable manifold) and then diverge away along a different line (unstable manifold). Specifically, we searched for trajectories that satisfy the following criteria. If the sequence of points is marked as $1,2,3 \ldots$, then we have the following.

(1) The point 1 is away from the identity line, and the point 2 lies close to the identity line.

(2) At least two subsequent points diverge away from point 2 along a straight line with a negative slope with magnitude greater than unity. The intersection of this line with the identity line is taken to be the location of the UFP.

(3) The points on the unstable manifold alternate on the two sides of the identity line (flip-saddle criterion).

(4) At least two such sequences of points, starting at different times, exhibit similar trajectories near the same UFP (recurrence criterion).

(5) The best-fit straight lines to the first points of all these recurrent sequences intersect the identity line near the UFP. This line, whose slope is usually close to zero (the magnitude of the slope must be less than unity), defines the stable manifold.

With short sequences (3-5 points), it is not possible to check with certainty that the sequence of points defining the unstable manifold diverge exponentially away from the UFP. This condition is approximately satisfied by most of the sequences obtained with the criteria listed above. The manifolds are determined through least-square fits to the corresponding points in all the recurrent sequences. If the stable manifold does not pass through the UFP, a straight line parallel to this line and passing through the UFP is taken as the stable manifold. An example of recurrent UPO-like trajectories found in network 1 is shown in Fig. 5 along with the computed stable and unstable manifolds. Another example from the same network may be found in Ref. [21].

After the determination of the UFPs and the corresponding stable and unstable manifolds, simulations of various control methods (chaos control, periodic pacing, demand pacing and anticontrol) around one selected UFP for each of the six networks were carried out. The chosen UFPs and the slopes of the manifolds used for these control applications are summarized in Table I. These control methods involve the generation of bursts at appropriately chosen times by the application of an external stimulation. An external stimulation was modeled in our simulations by a reduction of the inhibition strength $w$ from 0.6 to 0.24 for five passes.

For each of the control methods, we carried out simulations in which 5000 bursts are generated. A statistical summary of the results of these control simulations is provided in Table II. This table gives the values of the average IBI $\bar{T}$, the standard deviation $\sigma$ of the IBIs, and the percentage of IBIs above $\left(T_{n}>T^{*}+t_{s m}\right)$, below $\left(T_{n}<T^{*}-t_{s m}\right)$, and near $\left(T^{*}-t_{s m} \leqslant T_{n} \leqslant T^{*}+t_{s m}\right)$ the selected UFP $T^{*}$. The percentage (sgs) of IBIs generated by applications of the control stimulation is also listed for each of the control methods. 
TABLE I. The UFP $\left(T^{*}\right)$, the slope of the stable manifold $\left(m_{s}\right)$, and the slope of the unstable manifold $\left(m_{u s}\right)$ chosen for control in six networks. The percentages of IBIs above, below, and near the stable manifold (SM) during chaos control are given in columns 4 , 5 , and 6 , respectively.

\begin{tabular}{ccccccc}
\hline \hline No. & $T^{*}$ & $m_{s}$ & $m_{u s}$ & $\begin{array}{c}\text { Above } \\
\text { SM }\end{array}$ & $\begin{array}{c}\text { Below } \\
\text { SM }\end{array}$ & $\begin{array}{c} \pm t_{s m} \\
\text { of SM }\end{array}$ \\
\hline 1 & 345 & -0.041 & -1.0623 & 0.1 & 55.0 & 44.9 \\
2 & 1121 & -0.127 & -1.18 & 1.6 & 55.8 & 42.6 \\
3 & 823 & 0.097 & -1.54 & 0.6 & 56.6 & 42.8 \\
4 & 447 & 0.342 & -1.43 & 7.0 & 30.2 & 62.8 \\
5 & 1099 & -0.257 & -1.468 & 2.1 & 71.1 & 26.8 \\
6 & 937 & -0.476 & -1.683 & 0.0 & 65.5 & 34.5 \\
\hline \hline
\end{tabular}

\section{B. Chaos control}

The applied chaos-control method $[9,12]$ is a variant of the original technique proposed by Ott, Grebogi, and Yorke [10]. In this method, also referred to as the proportional perturbative feedback (PPF) method [27], artificial bursts are produced through timely stimulations that attempt to place the state point on the stable manifold of the UPO selected for control. Whenever a burst occurs, the current IBI $T_{n}$ is computed and the time at which the next burst should occur in order to place the state point on the stable manifold in the return map is calculated from the equation of the stable manifold. This time is $t_{n+1}+m_{s}\left(T_{n}-T^{*}\right)+T^{*}$, where $t_{n+1}$ is the time of occurrence of the last burst $\left(T_{n}=t_{n+1}-t_{n}\right)$ and $m_{s}$ is the slope of the stable manifold. An external stimulus is applied at this time if no natural burst occurs before its application. If a natural burst occurs prior to the application of the stimulus, then the time of application of the stimulus is recalculated using the value of the new IBI. In Fig. 6, two sequences of IBIs with such control from networks 1 and 4 are plotted. The control clearly increases the number of IBIs with values close to $T^{*}$. These control results are qualitatively similar to those reported by Schiff et al. for brain slices.

However, a close inspection of the control results shows that the control mechanism "works" mostly by preventing the occurrence of natural IBIs above the stable manifold. The external stimulus that attempts to place the state point on the stable manifold almost always produces a burst. This makes the occurrence of bursts with $T_{n+1}>m_{s}\left(T_{n}-T^{*}\right)+T^{*}$ extremely rare. The occurrence of spontaneous IBIs below the

TABLE II. Comparison of IBI statistics from six networks during no control (NC), chaos control (CC), periodic pacing (PP), and demand pacing (DP). The average IBI $\bar{T}$, the number of passes to generate 5000 IBIs, and the standard deviation $\sigma$ of the IBIs are given in columns 3, 4, and 5, respectively. Columns 6, 7, and 8 list the percentage of IBIs generated above, below, and near the UFP, respectively. The last column (sgs) lists the percentage of IBIs generated by external stimuli.

\begin{tabular}{|c|c|c|c|c|c|c|c|c|}
\hline No. & Type & $\begin{array}{c}\text { Duration } \\
\times 10^{5}\end{array}$ & $\bar{T}$ & $\sigma$ & $\begin{array}{c}\text { Above } \\
T^{*}\end{array}$ & $\begin{array}{c}\text { Below } \\
T^{*}\end{array}$ & $\begin{array}{c}\text { Around } \\
T^{*}\end{array}$ & sgs \\
\hline \multirow[t]{4}{*}{1} & $\mathrm{NC}$ & 18.442 & 369 & 297 & 34.3 & 53.8 & 11.9 & \\
\hline & $\mathrm{CC}$ & 12.575 & 251 & 100 & 0.1 & 54.4 & 45.5 & 38.5 \\
\hline & PP & 10.915 & 218 & 102 & 2.3 & 71.0 & 26.7 & 47.7 \\
\hline & DP & 12.322 & 246 & 99 & 0.1 & 56.1 & 43.8 & 37.4 \\
\hline \multirow[t]{4}{*}{2} & $\mathrm{NC}$ & 66.027 & 1320 & 1236 & 42.3 & 54.8 & 2.9 & \\
\hline & $\mathrm{CC}$ & 41.304 & 826 & 492 & 19.9 & 55.2 & 24.9 & 39.0 \\
\hline & PP & 32.075 & 641 & 397 & 1.7 & 74.5 & 23.8 & 32.7 \\
\hline & DP & 40.482 & 810 & 469 & 1.6 & 55.3 & 43.1 & 40.1 \\
\hline \multirow[t]{4}{*}{3} & $\mathrm{NC}$ & 44.16 & 883 & 816 & 38.2 & 57.2 & 4.6 & \\
\hline & $\mathrm{CC}$ & 27.561 & 551 & 290 & 0.6 & 73.1 & 26.3 & 40.0 \\
\hline & PP & 23.071 & 461 & 272 & 1.1 & 76.7 & 22.2 & 45.4 \\
\hline & DP & 27.964 & 559 & 283 & 0.5 & 59.6 & 39.9 & 36.7 \\
\hline \multirow[t]{4}{*}{4} & $\mathrm{NC}$ & 48.938 & 979 & 930 & 62.3 & 32.1 & 5.6 & \\
\hline & $\mathrm{CC}$ & 21.977 & 440 & 392 & 12.4 & 48.6 & 39.0 & 57.0 \\
\hline & PP & 17.876 & 358 & 186 & 7.3 & 48.9 & 43.8 & 50.1 \\
\hline & $\mathrm{DP}$ & 22.326 & 447 & 397 & 7.2 & 31.5 & 61.3 & 55.4 \\
\hline \multirow[t]{4}{*}{5} & $\mathrm{NC}$ & 47.191 & 944 & 898 & 28.9 & 68.3 & 2.8 & \\
\hline & $\mathrm{CC}$ & 35.945 & 719 & 468 & 19.4 & 71.0 & 9.6 & 24.6 \\
\hline & PP & 27.853 & 558 & 376 & 2.5 & 83.6 & 13.9 & 39.7 \\
\hline & $\mathrm{DP}$ & 34.966 & 699 & 442 & 2.8 & 67.7 & 29.4 & 26.9 \\
\hline \multirow[t]{4}{*}{6} & $\mathrm{NC}$ & 49.383 & 988 & 953 & 36.4 & 60.2 & 3.4 & \\
\hline & $\mathrm{CC}$ & 33.041 & 661 & 369 & 23.2 & 68.1 & 8.7 & 32.8 \\
\hline & PP & 25.477 & 510 & 303 & 0.4 & 78.5 & 21.1 & 49.2 \\
\hline & DP & 31.113 & 622 & 314 & 0.0 & 60.0 & 40.0 & 38.1 \\
\hline
\end{tabular}



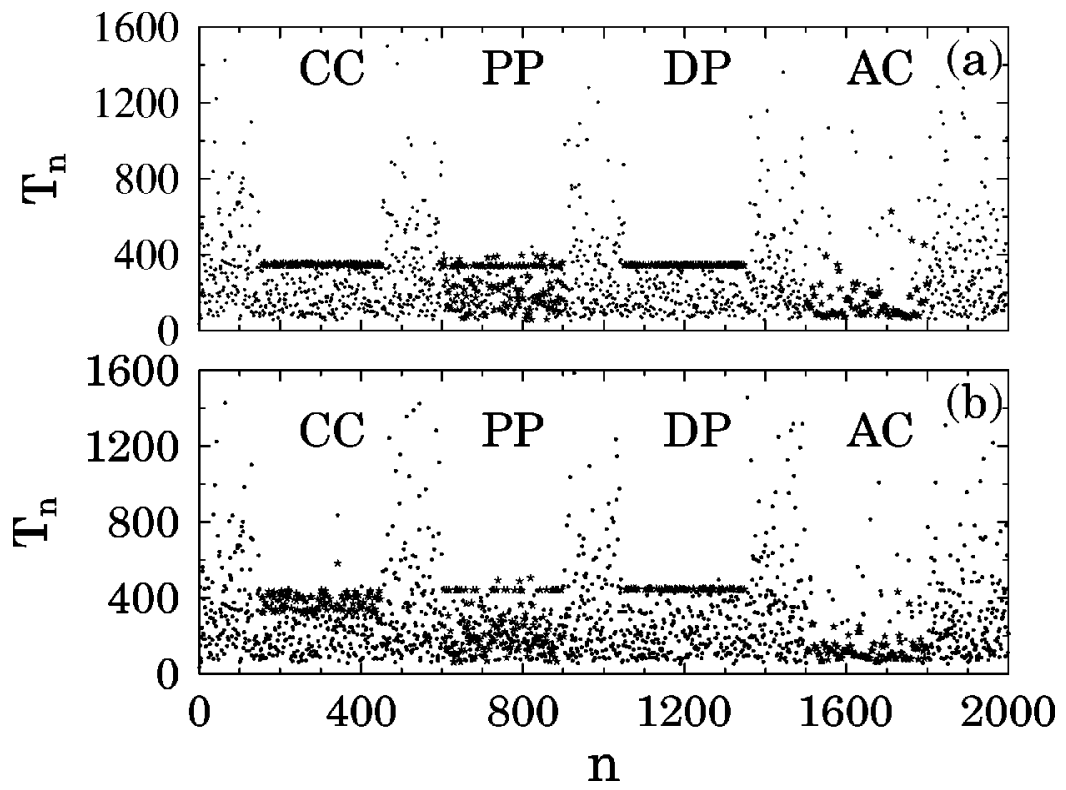

FIG. 6. Comparison of control methods. Results of chaos control (CC), periodic pacing (PP), demand pacing (DP), and anticontrol (AC) runs that produce $300 \mathrm{IBIs}$ each in (a) network 1 and (b) network 4 are shown. No control is applied during the intermediate periods of 150 IBIs. IBIs generated by the external stimuli are denoted by a different symbol (stars) to distinguish them from the spontaneous IBIs. stable manifold are not significantly affected by the application of the control. Consequently, the control procedure works better (i.e., the fraction of IBIs near $T^{*}$ increases) if $T^{*}$ is small and the slope of the stable manifold is close to zero. This is illustrated in Fig. 6 where the "quality" of control in network 4, for which both $T^{*}$ and $m_{s}$ are larger than those for network 1 (see Table I), is clearly found to be worse than that in network 1: the fraction of IBIs near $T^{*}$ is smaller, and the fraction of IBIs greater than $T^{*}$ is higher in network 4 (see Table II for the values of these fractions). The results of control are also better if the average IBI in the undisturbed network is large: this decreases the number of spontaneous IBIs lying below the stable manifold. All these characteristics of the control results may be found in the statistical summary given in Table II. These results are qualitatively similar to those reported in Ref. [12] for a stochastic single-neuron model.

In a truly deterministic chaotic system, intermittent applications of the external stimulus should lead to effective control. Once the state point is correctly placed close to the stable manifold by an external stimulus, a few subsequent points should continue to remain near the stable manifold without any external intervention. The trajectory would then deviate away, needing external intervention after some time to put the state point back in the vicinity of the stable manifold. In our simulations, intermittent applications of the control stimulus did not produce effective control. This is consistent with the purely stochastic nature of the bursting dynamics of our model. Intermittent control applications were also unsuccessful in the brain-slice experiment [9], although this may also be attributed to inaccuracies in the determination of the slope of the stable manifold from noisy (and possibly nonstationary) physiological data.

If the state point remains close to the stable manifold and approaches the UFP for some time after it has been placed there by a control stimulus (as it should in a system exhibiting deterministic chaos), then a majority of the IBIs found near the fixed point in the presence of control should occur naturally rather than through applications of the external stimulus. In our simulations where the underlying dynamics is stochastic, almost all the IBIs clustering around the fixed point value and giving the impression of successful control are generated by the control stimulus. This can be seen in Fig. 6 where the stimulus-generated IBIs and the naturally occurring ones are plotted with different symbols. As emphasized in Ref. [28], such an analysis is absolutely necessary for an unambiguous interpretation of the apparent success of chaos control found in the brain-slice experiment. Since the chaos control procedure attempts to put the system point on the stable manifold, a relevant quantity to consider is the fraction of IBIs found within $\pm t_{s m}$ of the stable manifold during the application of chaos control. Values of this quantity for the six networks are given in Table I. These values are close to (slightly higher than) the fractions of stimulusgenerated IBIs given in Table II. This confirms that nearly all points found near the stable manifold during chaos control are generated by the control stimulus.

As indicated in Table I, a small fraction of IBIs are found to lie above the stable manifold even when the control is on. This is due to rare failures of the external stimulus to generate a burst. Increasing the strength of the stimulus (i.e., increasing the amount by which $w$ is decreased during the application of the stimulus) improves the quality of control by decreasing the number of IBIs lying above the stable manifold. This is analogous to the improvement in control found in the brain-slice experiment when two pulses instead of one were used to generate a burst at the appropriate time.

Slutzky et al. [18] have recently reported the results of applications of chaos control methods to spontaneously bursting hippocampal slices similar to those studied in Ref. [9]. One of the control methods used by them is similar to ours, with the difference that in their experiment, the control stimulus to generate a burst was applied only when the distance of the state point from the selected UFP exceeded a suitably chosen "control radius" $R_{c}$. Large values of $R_{c}$ correspond to intermittent applications of the control stimulus, and this method reduces to the one used by us in the limit of very small values of $R_{c}$. Slutzky et al. studied the 
effects of changing the value of $R_{c}$ on the efficacy of control. They also kept track of the fraction of bursts generated by the control stimulus. They found that the quality of control, measured by the variance of the IBIs generated during control, increases as $R_{c}$ is decreased. However, decreasing $R_{c}$ also increased the fraction of stimulus-generated IBIs, indicating that the improvement of control was mostly due to the generation of many IBIs near $T^{*}$ by the external stimulus. This is similar to the results of our simulations. The values of $T^{*}$ and $m_{s}$ for the UPOs selected for control were not reported in Ref. [18]. It appears from the plots shown in this paper that good control was achieved only when $T^{*}$ is close to the lower limit of the IBI distribution and the value of $\left|m_{s}\right|$ is close to zero. As mentioned above, a very similar behavior was observed in our simulations of PPF control. Slutzky et al. have also reported the results of applications of an adaptive tracking method [29] that continuously refines the estimates of $T^{*}$ and $m_{s}$ during the application of control, and a new protocol called "state point forcing" that helps in determining the validity of fixed point estimates. Simulations of these methods for our network model are planned for the near future.

\section{Periodic pacing}

In periodic pacing, the network is stimulated at fixed intervals equal to $T^{*}$ irrespective of the occurrence of natural spikes. As a result, many artificial bursts at intervals equal to $T^{*}$ are generated, and naturally occurring bursts corresponding to IBIs larger than $T^{*}$ are eliminated. Periodic pacing also produces many new IBIs smaller than $T^{*}$-these are due to spontaneous bursts occurring between two successive applications of the control stimulus.

Two sequences of IBIs with periodic pacing from networks 1 and 4 are plotted In Fig. 6. From the density of points near $T^{*}$, it is evident that in network 1 periodic pacing is less effective than chaos control in increasing the periodicity of the bursts. The reverse is true in network 4 . The quantitative differences between the results obtained for the two networks may be found in Table II. These differences are mainly due to differences in the slopes of the stable manifolds. As discussed in the preceding section, chaos control works well in network 1 because both $T^{*}$ and $\left|m_{s}\right|$ are relatively small. Since $\left|m_{s}\right|$ is close to zero in this case, trying to place the state point on the stable manifold during chaos control is basically the same as periodic pacing, except that the external stimulation is not applied if a spontaneous burst occurs before its application. This eliminates the large number of small IBIs found in periodic pacing. In network 4, chaos control produces a large number of IBIs near the stable manifold, but only a small fraction of them lie near $T^{*}$ because $\left|m_{s}\right|$ is relatively large. So, if the fraction of IBIs near $T^{*}$ is taken to be the measure of the effectiveness of the control procedure, then periodic pacing works better than chaos control in this network.

It is clear from Fig. 6 that a large fraction of the IBIs found near $T^{*}$ during periodic pacing are generated by the external stimulus. In contrast to the behavior during chaos control, a large number of IBIs lower than $T^{*}$ are also gen- erated by the external stimulus. This is due to spontaneous bursts between two successive applications of the stimulus. A small number of IBIs with values larger than $T^{*}$ are also found. As noted above, these are due to occasional failures of the stimulus in producing the required burst.

These features are similar to the results of the brain-slice experiment [9]. However, some of the successful periodic pacing results in the brain-slice experiment, where the number of IBIs below $T^{*}$ are also apparently reduced, cannot be reproduced in our network simulation. Periodic pacing in the networks always increases the number of IBIs below $T^{*}$ (see Table II). In fact, the amount of time required to generate a fixed number of IBIs decreases (the value of the average IBI becomes smaller) whenever any form of control is applied to our networks. Applications of the external stimulus to generate bursts at appropriate times during control increases the number of bursts, but the naturally occurring bursts in our stochastic model are not affected by the control. As a result, the total number of bursts in a fixed period always increases during control. This is clear from the data shown in Table II. A convincing demonstration that the average IBI can be increased by the application of one of these control methods in brain-slice experiments would provide strong support to the claim $[9,16]$ of deterministic behavior in such systems.

\section{Demand pacing}

In demand pacing, the network is prevented from producing any IBI higher than the UFP by timely applications of the external stimulus. Given the occurrence of a burst at time $t_{n}$, the external stimulus is applied at time $t_{n}+T^{*}$ if no spontaneous burst occurs before its application. This procedure is essentially the same as the PPF chaos-control method described above if the slope of the stable manifold is close to zero. This similarity between PPF control and demand pacing has been noted in a recent experimental study [18]. For stable manifolds of larger slope, however, demand pacing is more effective than chaos control in increasing the periodicity of the bursts. Demand pacing always works better than periodic pacing by eliminating some of the short IBIs generated in periodic pacing through the occurrence of spontaneous bursts between two successive applications of the external stimulus. In all the networks, the number of IBIs near the fixed point in demand pacing is close to the sum of the number of stimulus-generated bursts and the number of spontaneous bursts expected near the fixed point in the undisturbed network during the period of application of control.

The results of applications of demand pacing to networks 1 and 4 are plotted in Fig. 6. The characteristics of demand pacing mentioned above are evident from this figure, and also from the quantitative results shown in Table II. These results are consistent with the underlying stochastic dynamics of the networks. Specifically, the increased effectiveness of demand pacing over chaos control when $\left|m_{s}\right|$ is large is expected only if the dynamics is stochastic. In truly chaotic dynamics, a chaos-control mechanism that makes use of the saddle structure near a candidate UPOs is expected to be qualitatively different from, and more effective than demand pacing which amounts to just cutting off the IBIs above the 
UFP. Therefore, if the bursting in brain slices were truly deterministic, then, irrespective of the slope of the stable manifold, chaos control should always be more effective than demand pacing in increasing the periodicity of the bursts. An experiment in which the effectivenesses of these two methods are compared would be very helpful in understanding the nature of the underlying dynamics and in establishing unambiguously the occurrence of chaos.

\section{E. Anticontrol}

Anticontrol methods [30-33] use the underlying deterministic dynamics to increase or preserve its complexity. Schiff et al. [9] tried to achieve this by applying PPF-type control for an appropriately chosen anticontrol line ("repellor" line) that passes through the selected UFP. They made the ad hoc choice of the repellor line to be the mirror image of the unstable manifold about the identity line. It was claimed that this choice effectively diverted the state point away from the UFP and increased the variability of IBIs. In the brain-slice experiment, anticontrol applications had limited success: only a small fraction of the attempts appeared to be effective in increasing the variability of the IBIs. Qualitatively similar results were obtained by Christini and Collins [12] in their simulations of a stochastic single-neuron model.

A proper assessment of the success of anticontrol requires a quantitative measure of its effectiveness in increasing the variability of the IBIs. No such measure was defined in Refs. [9] and [12]. We have defined two dimensionless quantities that may be used for this purpose. The first one is the ratio $\left(r_{1}\right)$ of the standard deviations of the IBIs recorded during an anticontrol run and during a run of the same duration without any control. Values of $r_{1}$ greater than unity would indicate success of anticontrol. A second quantity we have considered is the ratio $\left(r_{2}\right)$ of the standard deviation and the mean of the IBIs recorded during anticontrol. This quantity measures the scaled variability of the IBI distribution. Values of $r_{2}$ obtained from simulations of our networks in the absence of any control lie between 0.8 and 0.95 , the smaller values being obtained in networks with relatively small values of the mean IBI (see Table II). Deviations of these values from unity, the value expected for a Poisson distribution, are due to the small- $T$ cutoff in the IBI distribution. Substantially higher values of $r_{2}$ during the application of anticontrol would indicate its success.

Repellor lines obtained from the prescription of Schiff et al. [9] have negative slopes with magnitude smaller than unity. As a result, the control stimulus attempts to generate a large IBI after the occurrence of a small one, and vice versa. Also, stimulus-generated IBIs cannot exceed the value given by the point at which the repellor line intersects the $y$ axis. As noted above, this prescription for selecting the slope of the repellor line does not have any clear justification. A different choice was made by Christini and Collins [12] who took the repellor line to be the mirror image of the unstable manifold about a line passing through the UFP and perpendicular to the $x$ axis. Since the slope of the unstable manifold is always negative with magnitude larger than unity, the slope of such a repellor line is always positive and larger than unity. Such lines impose no upper bound on stimulusgenerated IBIs. A small IBI leads to a subsequent small IBI induced by the control stimulus, and a large IBI leads to a large one unless a natural burst occurs prior to the application of the control stimulus.

In the absence of any clear-cut prescription for choosing the slope $s$ of the repellor line, we have systematically studied the effectiveness of anticontrol for many different values of $s$. We found that if the number of IBIs recorded during an application of anticontrol is sufficiently large $\left(>10^{3}\right)$ to give reliable statistics, then the standard deviation of the IBIs during anticontrol is smaller than that for the undisturbed network $\left(r_{1}<1\right)$ for all choices of $s$. The reason for this behavior is the same as that mentioned above for the result that the average IBI obtained from our simulations in the presence of any form of control is smaller than that for the undisturbed network. In our stochastic networks, applications of the control stimulus generate additional bursts, but cannot prevent the occurrence of spontaneous bursts. Therefore, the number of relatively small IBIs can be increased, but the upper limit of the IBI-distribution cannot be extended beyond the value found in the same network without any control. However, in simulations of anticontrol over relatively small periods during which only a few hundred bursts are generated, "successful" anticontrol $\left(r_{1}>1\right)$ was observed in a few runs with large, negative values of $s$. This is purely due to statistical fluctuations: values of $r_{1}$ smaller than unity were obtained when the simulations were continued for longer periods. Similar to the experimental results, anticontrol failed in most cases even during these small-duration runs. Applications of anticontrol in brain slices for longer durations would be useful to determine whether the reported successes of anticontrol were due to the deterministic nature of the dynamics or due to inadequate statistics.

While this anticontrol method cannot increase the standard deviation of the IBIs in our stochastic networks, it can increase the relative variability $r_{2}$ of the IBI distribution for appropriate choices of the slope $s$ of the repellor line. We found that values of $s$ close to unity are most effective in producing large values of $r_{2}$. Repellor lines with $s$ slightly above unity do not impose any upper or lower bound on the stimulus-generated IBIs, and are definitely off the manifolds. Anticontrol with such values of $s$ produces many additional artificial IBIs of small magnitude. This decreases both the mean value and the standard deviation of the IBIs. The former is decreased more in comparison with the latter, so that the value of $r_{2}$ is increased. Our simulations with $s$ close to unity yield values of $r_{2}$ in the range 1.2-1.6. The results of two such applications of anticontrol over relatively small durations are shown in Fig. 6. These plots are visibly similar to those shown in Ref. [12]. We have also found that increasing the strength of the control stimulus increases the effectiveness of anticontrol, essentially by ensuring that the stimulus produces the desired burst. This is similar to the results reported in Ref. [9] for "double-pulse" control methods.

\section{F. Poisson simulations}

We have carried out Poisson simulations of all the control procedures described above. As mentioned in Sec. II C, these 


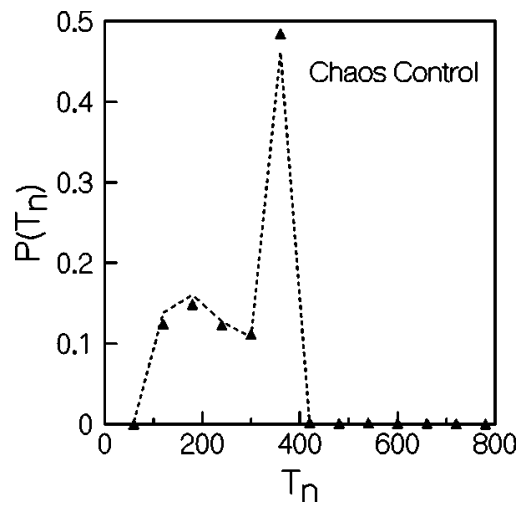

FIG. 7. Probability distribution of IBIs from network simulation (dotted line) and Poisson simulation (dark triangle) during chaos control in network 1 .

simulations are based on the assumption that the transition of the network to the high-activity state (i.e. the occurrence of a burst) is a stochastic process that has a probability $p$ of occurring at each time step. The values of $p$ for the six networks studied were computed from the results of network simulations. The additional ingredient required for simulating control methods is the process of generating bursts by applying an external stimulus. This was modeled by another independent stochastic process that has a high probability $p^{\prime}$ of occurrence at the time of application of the external stimulus. Values of $p^{\prime}\left(0.9 \leqslant p^{\prime} \leqslant 1.0\right)$ were obtained from network simulations where we kept track of the fraction of applied stimuli that produced bursts.

All the measurements made in the network simulations (the measured quantities are listed in Tables I and II) were repeated in our Poisson simulations. A comparison of the two sets of results for each network showed good agreement in all cases. Such comparisons for the probability distribution of the IBIs during chaos control, periodic pacing and demand pacing in network 1 are shown in Figs. 7, 8, and 9, respectively. These plots illustrate the close agreement between the results of network and Poisson simulations. Similar results for network 6 may be found in Ref. [21]. These results confirm the stochastic nature of the IBI time series generated in our network model.

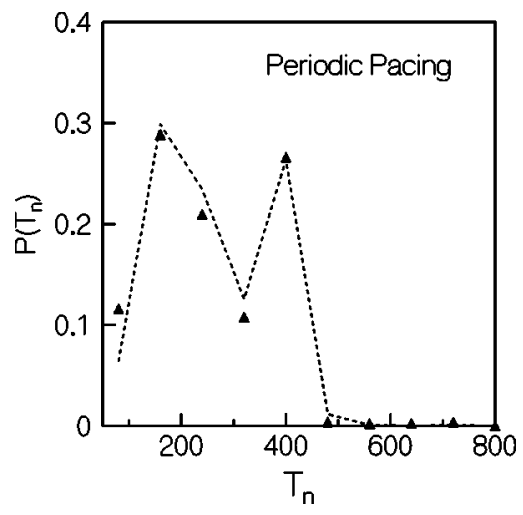

FIG. 8. Probability distribution of IBIs from network simulation (dotted line) and Poisson simulation (dark triangle) during periodic pacing in network 1 .

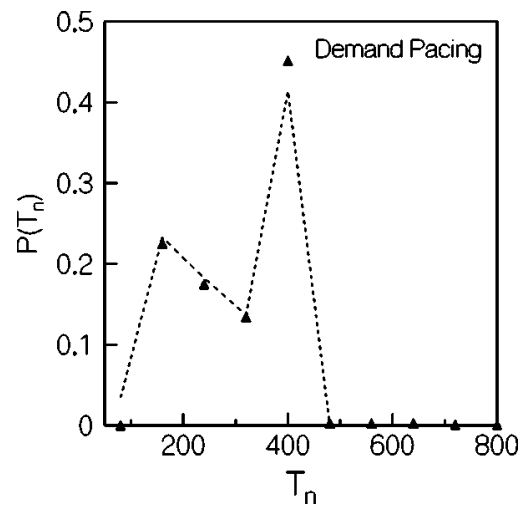

FIG. 9. Probability distribution of IBIs from network simulation (dotted line) and Poisson simulation (dark triangle) during demand pacing in network 1 .

\section{STATISTICAL SIGNIFICANCE OF UPOS}

As discussed in Secs. II and III, simulations of our stochastic neural network model qualitatively reproduce all the features found in the brain-slice experiment of Ref. [9]. This observation raises the following important question: do the results reported in Ref. [9] establish the occurrence of deterministic chaos in spontaneously bursting hippocampal slices, or alternatively, do these results reflect apparent deterministic features that appear purely as a matter of chance in a stochastic time series. This issue was first raised by Christini and Collins [12] who showed that simulations of a stochastic single-neuron model reproduce most of the results reported in Ref. [9].

More recently, a number of new methods have been proposed for locating UPOs in experimental time-series data and for assessing their statistical significance through surrogate analysis. So et al. [14] have proposed a method based on a dynamical transformation that utilizes the local dynamics to enhance the probability measure about distinct UPOs in state space. In subsequent work $[15,16]$, this method has been applied to analyze IBI datasets obtained from the brainslice experiment. These studies claim to have found UPOs in a statistically significant number of windowed datasets. Similar results have been found in a recent study [17] of hippocampal bursting under three different experimental conditions. In an analysis using the same dynamical transformation, but a different criterion for assessing the statistical significance of candidate UPOs, Van Quyen et al. [19] have reported detection of statistically significant UPOs in human epileptic activity. Pierson and Moss [13] have proposed a topological recurrence criterion for locating UPOs in noisy time-series data. Using this method in combination with surrogate analysis, Pei and Moss [34] have detected statistically significant UPOs in the dynamics of the crayfish caudal photoreceptor. Using the same method, de la Prida et al. [35] have found statistically significant UPOs in ISI data obtained from immature hippocampal networks.

To test the reliability of these statistical methods, and to check whether or not simulations of our model can reproduce some of the results obtained from their applications to experimental data, we have used these methods to analyze the stochastic IBI time series obtained from our network simu- 
lations. The results of these studies are described in this section.

\section{A. Method of So et al.}

We first consider period-1 orbits that correspond to fixed points in the first return map. In the method of So et al., such fixed points are detected by performing the following dynamical transformation [14] on the IBI time series $\left\{T_{n}\right\}$ :

$$
\hat{T}_{n}=\frac{T_{n+1}-s_{n}(k) T_{n}}{1-s_{n}(k)},
$$

where

$$
s_{n}(k)=\frac{T_{n+2}-T_{n+1}}{T_{n+1}-T_{n}}+k\left(T_{n+1}-T_{n}\right) .
$$

Under this transformation, most of the points in the linear region around a fixed point $T^{*}$ are mapped to the vicinity of $T^{*}$. Therefore, a histogram of the probability distribution $\rho(\hat{T})$ of the transformed time series $\left\{\hat{T}_{n}\right\}$ shows a sharp peak at each fixed point. Spurious peaks, which depend strongly on the parameter $k$, are eliminated by averaging the distribution over a large number of random values of $k$ obtained as $k=\kappa R$ where $R$ is a random number distributed uniformly in $[-1,1]$, and $\kappa$ is a parameter chosen to ensure that the two terms on the right-hand side of Eq. (7) have the same order of magnitude. The statistical significance of candidate UPOs corresponding to peaks of $\rho(\hat{T})$ is assessed from a comparison with the distribution obtained for a large number of carefully constructed, randomized versions (surrogates) of the original dataset.

In the analysis $[15,16]$ of the brain-slice data using this method, it was argued that in biological systems the parameters governing the underlying dynamics are not stationary in time. This argument contradicts to some extent the interpretation of the control results reported in Ref. [9], where it was claimed that candidate UPOs detected from a search during a "learning" period can be used to control the dynamics in subsequent time intervals of substantial duration. Nevertheless, assuming the system parameters to vary slowly in time, the IBI dataset obtained from the experiment was broken up into small overlapping [15] or nonoverlapping [16] windows of size $m$, which is chosen to be small enough to maintain stationarity and large enough to provide adequate statistics. The transformation of Eq. (6) was then applied to the data in each window to obtain the distribution $\rho(\hat{T})$.

Using many realizations of Gaussian-scaled phaseshuffled surrogates [36] (random shuffling that approximately preserve the power spectrum) of the original data in each window, the statistical probability that the observed peaks in the transformed $\rho(\hat{T})$ could be found in random surrogates was estimated. For each realization of the surrogate data, the same procedure was applied to calculate the distribution $\rho_{\text {sur }}(\hat{T})$. Then, from this collection of $\left\{\rho_{\text {sur }}(\hat{T})\right\}$, the ensemble-averaged distribution $\bar{\rho}_{\text {sur }}(\hat{T})$ was obtained.
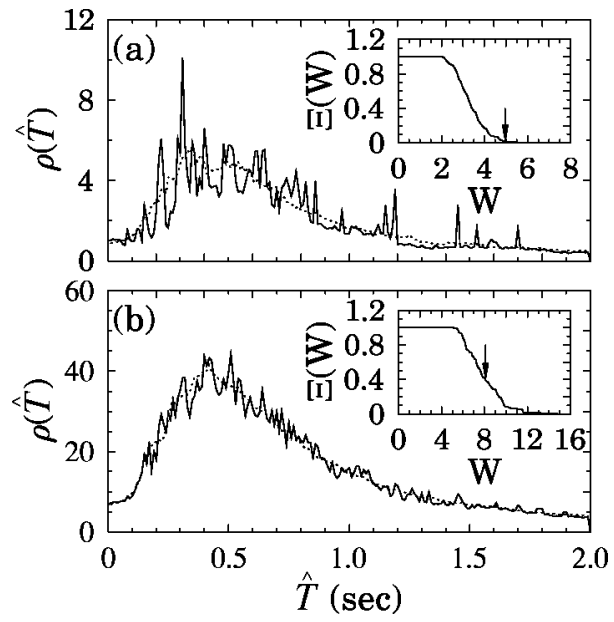

FIG. 10. Statistical significance of UPOs (method of So et al., see Sec. IV A) in windowed datasets. The plots show histograms for the probability distribution $\rho(\hat{T})$ (solid line) of the transformed series for the original dataset, and the average probability distribution $\bar{\rho}(\hat{T})$ (dotted line) of the transformed series for the surrogates. Results for a 512-IBI dataset and a 4096-IBI dataset are shown in panels (a) and (b), respectively. Inset: Plots of $\Xi(W)$, the fraction of surrogates with maximum deviation exceeding $W$ (see text). The maximum deviation $W_{\max }$ between $\rho(\hat{T})$ and $\bar{\rho}(\hat{T})$ occurs at $\hat{T}$ $=0.31 \mathrm{~s}\left(W_{\max }=4.97\right)$ for $(\mathrm{a})$, and at $\hat{T}=0.51 \mathrm{~s}\left(W_{\max }=8.11\right)$ for (b). Arrows at $W_{\max }$ indicate the statistical significance level $\Xi\left(W_{\max }\right)$ (see Sec. IV A).

Fluctuations of the distribution for an individual realization of the surrogates from the ensemble average were measured by

$$
w(\hat{T})=\rho_{\text {sur }}(\hat{T})-\bar{\rho}_{\text {sur }}(\hat{T}) .
$$

For each surrogate, the maximum deviation $W$ $=\max [w(\hat{T})]$ was measured, and the fraction $\Xi\left(W^{\prime}\right)$ of surrogates with maximum deviation $W>W^{\prime}$ was found using a large number of surrogates. Then, the statistical significance of a candidate UPO at $\hat{T}^{*}$ was estimated from the value of $\Xi\left(W^{*}\right)$ where $W^{*}=\rho\left(\hat{T}^{*}\right)-\bar{\rho}_{\text {sur }}\left(\hat{T}^{*}\right)$. Specifically, the statistical confidence level for the detection of an UPO was estimated to be $\left[1-\Xi\left(W^{*}\right)\right] \times 100 \%$.

We have carried out the same analysis using windowed datasets obtained from our network simulations. We scaled the IBI data to match the experimental data used in the analysis of Ref. [15]. In that work, 1834 ISIs collected over $25 \mathrm{~min}$ were analyzed for the detection of UPOs. In network 1 , the same number of IBIs are generated in 685409 passes. We, therefore, scaled our time unit by setting one pass equal to $0.0022 \mathrm{~s}$. In our analysis, we used 100 surrogates, took $\kappa=5.0$, and averaged the distribution over 500 values of the random number $R$. Representative results of this analysis are shown in Fig. 10. We found that this method successfully rejects the UPO-like trajectories found in the first return maps of the IBI time series obtained from our network simulations only if the dataset used in the analysis is sufficiently large $(m \geqslant 2048)$. Our results for a dataset with $m=4096$, 
TABLE III. Statistical significance of UPOs in windowed datasets from ten networks, according to the method of So et al. (see Sec. IV A). The number of IBIs in each nonoverlapping window and the percentage of networks showing statistically significant UPOs are given in columns 1 and 2, respectively. The total number of windows used in the analysis and the percentage of windows showing statistically significant UPOs are given in columns 3 and 4 , respectively.

\begin{tabular}{lccc}
\hline \hline Window size & $\begin{array}{c}\text { \% significant } \\
\text { networks }\end{array}$ & No. of windows & $\begin{array}{c}\% \text { significant } \\
\text { windows }\end{array}$ \\
\hline 32 & 80 & 544 & 8.82 \\
64 & 100 & 272 & 10.29 \\
128 & 50 & 136 & 8.82 \\
\hline \hline
\end{tabular}

obtained from simulations of network 1, are shown in Fig. 10(b). The maximum value of the deviation $W=\rho(\hat{T})$ $-\bar{\rho}_{\text {sur }}(\hat{T})$ is found to be $W_{\max }=8.11$, which occurs at $\hat{T}$ $=0.51$. However, the fraction of surrogates with maximum deviation exceeding this value is fairly large: $\Xi\left(W_{\max }\right)$ $\simeq 0.39$, as shown by the arrow in the inset of Fig. 10(b). This means that this candidate UPO should not be considered statistically significant at the confidence level of $95 \%$ used in the analysis of Ref. [16]. In a similar analysis carried out for 35 datasets with $m \geqslant 2048$, we found a statistically significant UPO in only one dataset with $m=2048$. Thus, this surrogate analysis recognizes our IBI time series to be stochastic if a sufficiently large dataset is used in the analysis.

However, the same analysis leads to false detections of "statistically significant" (at the 95\% confidence level) UPOs in a substantial fraction of windowed datasets if $m$ is small. The results for a window with $m=512$, obtained from simulations of network 1 and showing the presence of a "statistically significant" UPO, are plotted in Fig. 10(a). In this case, the probability of finding the peak $\left(W_{\max }=4.97\right.$ at $\hat{T}$ $=0.31$ ) in the surrogates is very low, around $1 \%$, as indicated by the arrow in the inset of the figure, leading to the false identification of a statistically significant UPO at $95 \%$ confidence level. A similar plot for a $m=128$ dataset may be found in Ref. [21].

We have carried out a systematic analysis for different values of $m$ and observed that the criteria adopted in Refs. [15] and [16] fail to recognize our IBI datasets as stochastic in a significant fraction of trials if $m$ is small. Results of this analysis, carried out for IBI datasets collected from ten networks, are summarized in Table III. The total number of nonoverlapping windows used in the analysis for each window size $m$ is listed in the third column of the table. The second column lists the percentage of networks with at least one window with a statistically significant UPO at $95 \%$ confidence level, and the fourth column lists the percentage $f$ of windows (combined from all networks) with statistically significant UPOs. These results are quite similar to those reported in Ref. [16] for extracellular brain-slice data at relatively low potassium levels.

A similar analysis for a two-dimensional delay embedding of the IBI time series obtained from the brain-slice experi- ment was performed in Ref. [15]. We have carried out the same analysis using simulation data obtained for network 1 . In our analysis, we scaled the IBI data to match the time scale of the experiment, used parameter values identical to those in Ref. [15] ( $\kappa=5.0,500$ values of $R$, and 100 surrogates), and considered a large number of overlapping and nonoverlapping windows of different size. The results of this analysis are very similar to those described above. For example, we found "statistically significant" UPOs in 75 out of 1024 nonoverlapping 32-IBI windows. The values of $f$ were found to vary between $1.6 \%$ and $9.4 \%$ as the window size $m$ was varied between 32 and 1024, and no UPO was found for $m \geqslant 2048$. In Ref. [15], the results of the surrogate analysis for the detection UPOs in overlapping 128-IBI windows were presented in the form of a gray-scale plot from which it is difficult to extract quantitative information such as the value of $f$. For this reason, we could not compare our results directly with those of Ref. [15].

The method of Ref. [14] can be extended [15] to the detection of orbits of period higher than 1. Using this method, So et al. [16] have detected statistically significant UPOs of higher period in intracellular data obtained from brain slices. Since intracellular recordings reflect a combination of collective and single-neuron dynamics, our network with binary neurons is not appropriate for the modeling of such data. Nevertheless, we have carried out a simple analysis for the detection of period-2 orbits in the simulation data for network 1 . Since a period-2 orbit corresponds to a fixed point of the second iterate of the dynamics, we considered the time series $T_{1}, T_{3}, T_{5}, \ldots, T_{m-1}\left(T_{2}, T_{4}, T_{6}, \ldots, T_{m}\right)$ consisting of only the odd-numbered (even-numbered) terms in a $m$-IBI window ( $m$ even) of the original time series, in our analysis for the detection of period- 2 orbits. The method described above for the detection of statistically significant fixed points was then applied separately to the odd- and even-numbered datasets. If a period- 2 orbit is present in the $m$-IBI window of the original dataset, then this calculation should show two statistically significant fixed points in both the odd- and even-numbered datasets, and the locations of these fixed points should be nearly the same in the two datasets. We have confirmed that this method succeeds in detecting period-2 UPOs in the Henon map.

Since the IBIs in our stochastic time series are independent random variables, the time series obtained by retaining only even- or odd-numbered terms are statistically identical to the original one. We, therefore, expect that a period-1 analysis for the even- and odd-numbered datasets would yield results that are very similar to those obtained in our analysis for period-1 orbits in the original dataset. In our original period-1 analysis, we found very few windows with more than one statistically significant UPOs. Further, since the even- and odd-numbered datasets are statistically independent, it is highly improbable that both of them would exhibit UPOs at approximately the same locations. So, the probability that the even- and odd-numbered datasets would each exhibit two statistically significant UPOs at approximately the same locations is expected to be very small for our stochastic time series. The results of our analysis are consistent with this expectation. In our analysis of a 32768 - 
IBI time series from network 1 with window sizes varying from 128 to 8192, we did not find any window in which the criteria mentioned above for the detection of period-2 orbits were satisfied. As expected, the fraction $f$ of windows showing statistically significant period-1 orbits was approximately the same for even- and odd-numbered datasets, and this number was close to that obtained in our original period-1 analysis. However, very few windows showed two period-1 orbits, and the results obtained for the even-numbered data from a particular window did not match the results for the odd-numbered data from the same window. These results confirm the stochastic nature of our IBI time series. It would be interesting to carry out a similar analysis for the brainslice data.

In Ref. [16], the values of $f$ obtained from the analysis of intracellular data are found to be significantly higher than those calculated from the data obtained from extracellular recordings. This may be due to the following reason. The extracellular data reflect the collective dynamics of the network, whereas intracellular recordings can resolve the "spikes" that occur during the bursting of the neuron from which the recordings are made. Therefore, extracellular recordings provide information about the times of occurrence of population bursts, while the time series obtained from intracellular recordings is one of interspike intervals (ISIs), which reflect a combination of collective and single-neuron dynamics. This difference is clear from the characteristic time scales [16] of these two kinds of data: the typical IBI in the extracellular data is of the order of $1 \mathrm{~s}$, whereas the typical ISI in the intracellular data is much smaller, of the order of $0.05 \mathrm{~s}$. Given these facts, the larger values of $f$ obtained in the analysis of the intracellular data may be interpreted as evidence for determinism in the spiking dynamics of individual neurons. This interpretation is supported by the results of de la Prida et al. [35], who carried out a careful surrogate analysis of intracellular data recorded from immature hippocampal networks. They found that the IBIs in their time series are purely stochastic, whereas the spikes that form the intrinsic structure of individual bursts show evidence for deterministic dynamics. These results suggest that determinism and stochasticity need not be mutually exclusive- the same system may show both kinds of dynamics at different levels and time scales. The dynamics of our networks illustrates the same point: the time evolution of our network in the low- and high-activity states is mostly deterministic, whereas transitions between these two states are stochastic.

Since the confidence level for the detection of statistically significant UPOs was set at $95 \%$ in our calculations, values of $f$ for the stochastic IBI data obtained from simulations of our network model are expected to be near 5\%. Our results for datasets with $m \geqslant 2048$ are consistent with this expectation. The significantly higher values of $f$ (near $10 \%$, as shown in Table III) obtained from our analysis of windowed datasets with $m \leqslant 128$ must then be attributed to insufficient sampling. The similarity between our results and those reported in Ref. [16] for the extracellular brain-slice data suggests that the relatively large values of $f$ obtained in Ref. [16] may also be due to insufficient sampling. It would be inter- esting to check this possibility by carrying out a surrogate analysis of the brain-slice data using windows of larger size. A reduction of the values of $f$ with increasing $m$ would suggest stochastic behavior. However, a similar trend would also be found if the dynamics of brain slices is nonstationary. So, it would be difficult to distinguish between stochasticity and nonstationarity from an analysis of this kind. This appears to be a drawback of this method of detecting UPOs in experimental time-series data. Another possible source of insufficient statistics in the analysis [16] of the brain-slice data is the relatively small number (between 33 and 71) of windows. Our studies show that the difference between the calculated and expected values of the fraction $f$ of windows showing statistically significant UPOs decreases as the number of windows used in the analysis is increased.

A recent study [17] has reported large values of $f$ (between $12.5 \%$ and $42.1 \%$ ) obtained from analysis of windowed brain-slice data. In this work, IBI data were recorded under three different experimental conditions: high- $\left[\mathrm{K}^{+}\right]$, low- $\left[\mathrm{Mg}^{2+}\right]$, and the presence of $\mathrm{GABA}_{A}$-receptor antagonists. The method of So et al. [15] with a two-dimensional delay embedding was applied to overlapping 256-IBI windows. The reported values of the fraction of experiments showing at least one window with a statistically significant UPO are similar to those obtained in our simulations. However, the values of $f$ are considerably larger. The statistical significance of the results obtained in the low- $\left[\mathrm{Mg}^{2+}\right]$ and $\mathrm{GABA}_{A}$-antagonist experiments is questionable because a small number (16 and 19, respectively) of windows were used in these experiments. In a dataset of 256 nonoverlapping 32-IBI windows obtained from simulations of network 1 , we found two separate 19 -window and 16-window segments for which the values of $f$ are $42.1 \%$ and $31.3 \%$, respectively, although the value of $f$ obtained for the full 256window dataset is much smaller $(10.5 \%)$. Short segments of $\sim 20$ windows with similar values of $f$ were also found in other datasets. Thus, our model can produce such large values of $f$ if a small number of windows is considered. However, we did not find any segment of 60 or more windows for which the value of $f$ is more than about $15 \%$. So, it is unlikely that our model would reproduce the period- 1 result of Ref. [17] for high- $\left[\mathrm{K}^{+}\right](f=29 \%$ for 62 windows $)$ and the result of Ref. [16] for $10.5 \mathrm{mM} \mathrm{K}^{+}(f=21 \%$ for 67 windows). More data to ascertain the statistical significance of these experimental results would be very useful.

\section{B. Method of Van Quyen et al.}

Van Quyen et al. [19] used a similar method to analyze ISI data obtained from human epileptic activity. We have carried out the same analysis for our simulation data. To repeat their method as closely as possible, the IBI data from network 1 were rescaled so that the range over which the IBIs vary remains approximately the same as that in the experimental data. The range of the experimental data is about $250 \mathrm{~ms}$, whereas that for the IBI data from network 1 is about 1000 passes. So, one pass in the network dynamics was assumed to be equal to $0.25 \mathrm{~ms}$. Other parameters used in our analysis, such as bin sizes for histograms, the radius of 


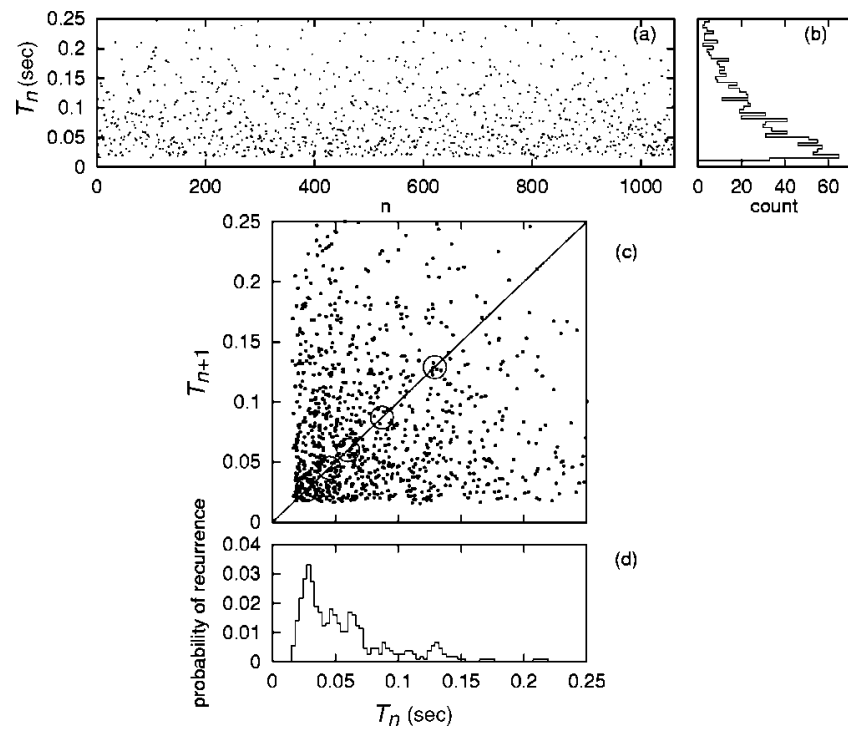

FIG. 11. Probability of recurrence of IBIs, according to the method of van Quyen et al. (see Sec. IV B). Panel (a): 1024 IBIs $\left(T_{n}\right)$ from network 1, rescaled to match the time scale of the experiment. Panel (b): Distribution of the IBIs. Panel (c): Return map with clustering of points (recurrence) along the identity line, marked with circles of radius $8 \mathrm{~ms}$. Panel (d): the probability density of the points along the diagonal.

circles on the identity line (see below) and the number of surrogates, were maintained at the values used by Van Quyen et al.

We used a dataset with $m=1024$ IBIs (this number is close to 1062 , the number of points in the dataset used in the analysis of Ref. [19]) obtained from simulations of network 1 in our analysis. The IBI dataset $\left\{T_{n}\right\}, n=1,2, \ldots, 1024$, used in our analysis and a histogram (bin size $=5 \mathrm{~ms}$ ) of the distribution of the IBIs are shown in Figs. 11(a) and 11(b). Our IBI data exhibit a Poisson distribution which is different from the nearly Gaussian distribution found in Ref. [19]. The density of points within a small circle of radius $8 \mathrm{~ms}$ around a point $T_{n}$ on the diagonal line in the return map provides a crude measure of the probability of successive IBIs to remain near the same $T_{n}$ [19]. A plot of this density (probability of recurrence) as a function of $T_{n}$ is shown in Fig. 11(d). Five main peaks in the recurrence probability, at $T_{1}=0.027 \mathrm{~s}$, $T_{2}=0.045 \mathrm{~s}, T_{3}=0.06 \mathrm{~s}, T_{4}=0.087 \mathrm{~s}$, and $T_{5}=0.125 \mathrm{~s}$, are found. The locations of these peaks in the return map are indicated by circles in Fig. 11(c). This inhomogeneous clustering of points in the return map is qualitatively similar to that observed in Ref. [19]. Therefore, the interpretation [19] of this clustering as a significant indication of correlation of successive IBIs may not be correct.

Since our IBIs follow a Poisson distribution, we could not use one of the methods used in Ref. [19] to construct the surrogate datasets. We considered three types of random surrogates that preserve the distribution of the IBIs. These are as follows.

(1) Gaussian-scaled phase-shuffle [36]. Random shuffling of the dataset in a manner that approximately preserves the power spectrum. This is the same as the method used to
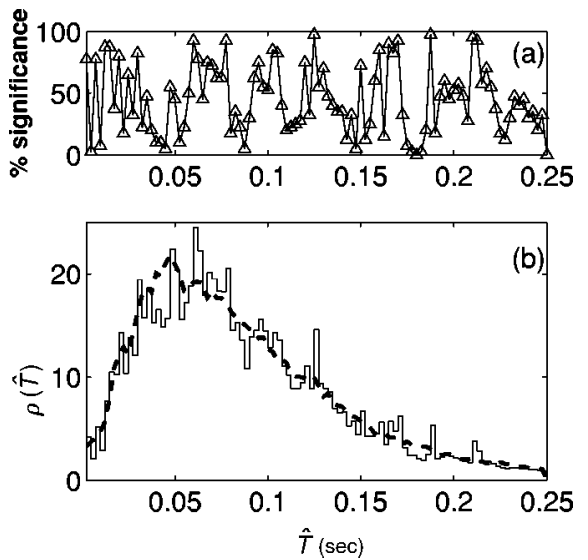

FIG. 12. Statistical significance of candidate UPOs near the peaks in Fig. 11(d). The solid line in panel (b) shows the histogram of $\rho(\hat{T})$ and the dashed line shows the average of $\rho_{\text {sur }}(\hat{T})$ obtained from 39 surrogates. The upper panel (a) shows the degree of statistical confidence in the detection of UPOs near the location of the peaks. The confidence level is defined as $100(1-P) \%$ with $P$ determined from a Monte Carlo test [Eq. (9)].

generate surrogates of type II in Ref. [19].

(2) Random shuffle. Simple random shuffling of the IBI dataset. This maintains the distribution but destroys correlations.

(3) Poisson simulation. Surrogate datasets generated from simulations of a Poisson process, as described in Sec. II.

The transformation of Eq. (6), with $\kappa=5$ and 500 values of $R$, was then applied to the original dataset and the surrogates, and the probability distribution $\rho(\hat{T})$ for the original data and the distributions $\rho_{\text {sur }}(\hat{T})$ for each of the surrogates were calculated. Following Ref. [19], we considered 39 different realizations of each type of surrogate data. The statistical significance of a candidate UPO corresponding to a peak of $\rho(\hat{T})$ at $\hat{T}=T^{*}$ was estimated by a one-tailed Monte Carlo test [19]:

$$
P=\frac{\left[\text { number of cases }\left\{\rho_{\text {sur }}\left(T^{*}\right) \geqslant \rho\left(T^{*}\right)\right\}\right]+1}{(\text { number of surrogates })+1} .
$$

Van Quyen et al. considered peaks with $P=0.025$, i.e., $\rho_{\text {sur }}\left(T^{*}\right)<\rho\left(T^{*}\right)$ for all 39 surrogates, to be statistically significant.

The results of our analysis for surrogates of type 1 are plotted in Fig. 12. The lower panel shows histograms of $\rho(\hat{T})$ and the average of $\rho_{\text {sur }}(\hat{T})$. The upper panel shows the degree of statistical confidence in the detection of UPOs. This is defined as $100(1-P) \%$. This analysis finds the peaks at $T_{5}$ to be statistically significant according to the criterion (97.5\% confidence level) adopted by Van Quyen et al. for all three types of surrogates. It is important to note that the remaining four peaks are found to be spurious in spite of the high weightage of these peaks in Fig. 11(d) and large clustering of points in this region of the return map [Fig. 11(c)]. In the surrogate analysis, peaks at $T=0.125 \mathrm{~s}$, $0.1875 \mathrm{~s}$ for surrogate type $1, T=0.015 \mathrm{~s}, 0.125 \mathrm{~s}, 0.1875 \mathrm{~s}$, 


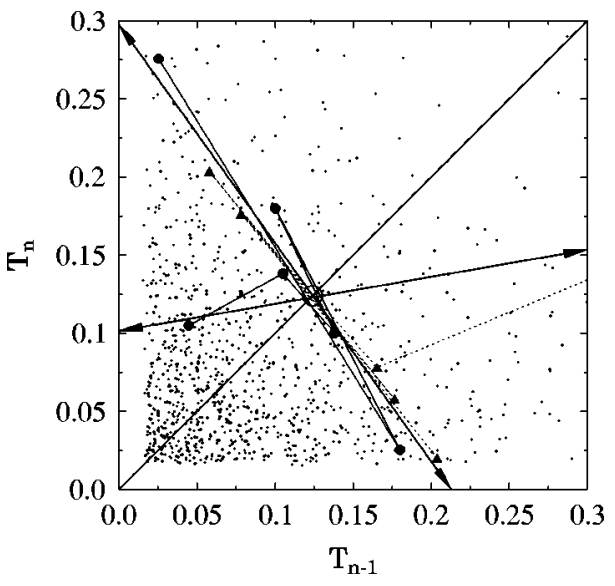

FIG. 13. Detection of UPO-like trajectories around the "statistically significant" UFP at $T_{5}=0.125 \mathrm{~s}$ (see Fig. 12).

$0.21 \mathrm{~s}$ for surrogate type 2 and $T=0.015 \mathrm{~s}, 0.125 \mathrm{~s}, 0.21 \mathrm{~s}$ for surrogate type 3 were found to be statistically significant at $97.5 \%$ confidence level. The peaks at $T=0.1875 \mathrm{~s}, 0.21 \mathrm{~s}$, found to be statistically significant for two of the three types of surrogates, are probably irrelevant, as they are in the tail of the distribution and no clustering of points on the identity line in the return map [Fig. 11(c)] is found around these two points.

Following Ref. [19], we further examined the significance of the apparent UPO at $T_{5}$ by looking for recurrent UPO-like trajectories around this point in the first return map. As shown in Fig. 13, such trajectories are indeed present in the IBI dataset. The "recurrent UPO" near $T_{5}$ has a stable manifold, $y=0.1735 x+0.1015$, and an unstable manifold, $y=-1.4 x+0.2978$. These are indicated in the figure by lines with arrows.

Surprisingly, this analysis fails to reject all UPO-like trajectories in our stochastic IBI dataset as spurious. Rather, the results are quantitatively similar to those of Ref. [19]. This clearly shows that the methods and criteria used by Van Quyen et al. are not completely reliable for identifying true UPOs. Therefore, some of the apparent UPOs found by them in human epileptic activity may not be real. The false positive results obtained in this analysis may be due to the small size of the dataset. When a larger dataset containing 8192 IBIs from the same network simulation was analyzed, significantly different results with no prominent peaks in the distribution $\rho(\hat{T})$ were obtained. The use of a small number of surrogates may be another reason for the failure of this method to identify our dataset as stochastic.

\section{Topological recurrence criterion}

In this method $[13,34]$, the number of UPO-like trajectories in the first return map of the original dataset is computed and compared with the values of the same quantity obtained for surrogates of the original dataset. The following criteria are used for identifying UPO-like trajectories.

Level 0 . There must be three sequential points that approach the identity line at successively decreasing perpendicular distances, followed by three sequential points that
TABLE IV. Results of surrogate analysis of IBI datasets from network 1, using the topological recurrence criterion (see Sec. IV C). Columns 1-7 list, respectively, the size of the dataset $m$ (number of IBIs), the number of Gaussian-scaled phase-shuffled surrogates used in the analysis, the number $N$ of UPO-like trajectories (encounters) found in the original dataset, the average number $\left\langle N_{s}\right\rangle$ of encounters found in the surrogates, the standard deviation $\sigma_{s}$ of the number of encounters found in the surrogates, the statistical measure $K=\left(N-\left\langle N_{s}\right\rangle\right) / \sigma_{s}$, and the maximum value $N_{\max }$ of the number of encounters found in the surrogates.

\begin{tabular}{rcccccc}
\hline \hline \multicolumn{1}{c}{$m$} & No. of surrogates & $N$ & $\left\langle N_{s}\right\rangle$ & $\sigma_{s}$ & $K$ & $N_{\max }$ \\
\hline 64 & 100 & 1 & 0.56 & 0.82 & 0.54 & 3 \\
128 & 100 & 2 & 1.34 & 1.02 & 0.65 & 4 \\
256 & 100 & 2 & 2.41 & 1.72 & -0.24 & 7 \\
512 & 100 & 3 & 4.29 & 2.07 & -0.62 & 10 \\
1024 & 100 & 8 & 8.52 & 3.18 & -0.16 & 16 \\
2048 & 100 & 18 & 16.87 & 3.97 & 0.28 & 25 \\
4096 & 100 & 35 & 34.72 & 6.44 & 0.04 & 53 \\
8192 & 100 & 80 & 72.67 & 8.73 & 0.84 & 96 \\
\hline \hline
\end{tabular}

depart at successively increasing distances. The third point is common to both sequences.

Level 1. A straight-line fit to the three approaching points must have a slope $m_{s}$ with $-1 \leqslant m_{s}<0$ and a straight-line fit to the three departing points must have a slope $m_{u s}$ with $-\infty<m_{u s}<-1$.

Level 2. The distance of the point of intersection of these two straight lines from the identity line must be smaller than half of the mean of the distances of the five points in the approaching and departing sequences.

We have analyzed IBI datasets from ten networks for "encounters" that satisfy the criteria mentioned above. Let $N$ be the number of encounters detected for a dataset. Using the same criteria, encounters are searched for in 100 Gaussianscaled phase-shuffled surrogates of the original dataset. Then, the statistical measure $K=\left(N-\left\langle N_{s}\right\rangle\right) / \sigma_{s}$ is computed, where $\left\langle N_{s}\right\rangle$ is the mean and $\sigma_{s}$ is the standard deviation of the number of encounters found in the surrogate datasets. For Gaussian statistics, $K \geqslant 2$ represents a statistically significant detection of the presence of UPOs with a confidence level greater than $95 \%$.

This surrogate analysis successfully identifies all the IBI datasets of varying lengths from the ten networks as stochastic, i.e., statistically significant UPOs are not found. The value of $K$ is always close to zero and sometimes negative for large datasets. For a few datasets of small length $(m$ $\leqslant 1024$ ), values of $K$ greater than 1 are found, but they are not statistically significant at the $95 \%$ confidence level. For some of the datasets, we also calculated the maximum value, $N_{\text {max }}$, of the number of encounters found in the surrogates. In all these cases, the number of encounters $N$ in the original dataset was found to be smaller than $N_{\max }$. Typical results obtained for network 1 are shown in Table IV. Similar results were obtained in an analysis of datasets obtained from Poisson simulations. 
We have also carried out a systematic analysis of the percentage $f$ of windows showing statistically significant UPOs according to this criterion. Using a dataset with 32768 IBIs obtained from simulations of network 1 , we found that the values of $f$ for different window sizes $(64 \leqslant m \leqslant 2048)$ lie between 0 and 6.3. These results are consistent with the expectation that about $5 \%$ of the windows of our stochastic IBI data should show statistically significant UPOs at $95 \%$ confidence level.

We, therefore, conclude that this method correctly identifies the datasets from our network and Poisson simulations as stochastic. However, we were surprised to find that this method also identifies certain datasets of small length, generated from the logistic map in the chaotic regime, as stochastic. For example, this method showed the presence of statistically significant UPOs in only 5 of 32 datasets of length 1024 generated from the logistic map with $a=3.99$. This observation argues against a complete reliability of this method. If the probability that a deterministic system visits the neighborhood of a particular UPO during the time span of the dataset being analyzed is comparable to or smaller than the probability of purely accidental appearance of UPOlike trajectories in a surrogate of the original dataset, then this method would wrongly identify the original data as stochastic. Since the probability of a deterministic system visiting the neighborhood of a UPO can, in general, be quite small (the logistic map is an example of this), this method would not work in all cases. However, the error that this method would produce in such cases is in a direction that is opposite to that of the other methods of surrogate data analysis we have considered. This method may identify a deterministic time series as a stochastic one, but it is unlikely to identify a stochastic time series as a deterministic one. The other methods we have considered lead to the (incorrect) conclusion that our stochastic IBI series has some characteristics of a deterministic one.

\section{SUMMARY AND DISCUSSION}

The main result of our investigation is the demonstration that simulations of our neural network model reproduce most of the features found in brain-slice experiments $[9,18]$, and in surrogate analysis [15-17] of the data obtained from these experiments. This result suggests that our model is an appropriate one for describing spontaneous population bursting in hippocampal slices. We have also shown that the variability of the IBIs in our network model is purely stochastic in origin. This casts serious doubt on the conclusions reached in Refs. [9,15-18] about signatures of deterministic chaos in the underlying dynamics of brain slices. Rather, our work strongly suggests that the bursting dynamics is well described by "bistability" with stochastic transition between a low-activity (normal) attractor and a high-activity (epileptic) attractor of the underlying neuronal network. The stochasticity of the IBIs in our simulations is a consequence of the use of random sequential updating. We believe that this updating scheme is the most "physical" one for describing the behavior of brain slices, because any external "clocklike" mechanism that may synchronize or order in time the firing of the neurons is not expected to be present in such in vitro systems. However, this may not be ruled out in in vivo systems, where the region of bursting activity continuously receives inputs from many other regions of the living brain [37].

There exist other results that support the conclusion that the bursting dynamics of brain slices is stochastic. The spontaneous bursting behavior of rat hippocampal slices, prepared in exactly the same way as the ones studied in the experiment of Schiff et al. [9], were tested for signatures of determinism in Ref. [25]. Three different tests were conducted for each of six slices. Out of the 18 tests, only one showed a statistically significant positive signature of determinism. In intracellular recordings from immature hippocampal networks [35], the IBIs were found to be purely random, following a Poisson distribution. Slutzky et al. [17] did not find any evidence of determinism in their "expansion rate analysis" of IBI data from hippocampal slices. A physiologically realistic computer model [38] of synchronized epileptiform bursts induced by high potassium concentration in rat hippocampal slices had to include stochasticity in the form of spontaneous excitatory postsynaptic potentials modeled by an independent Poisson process for each pyramidal cell. This is similar to the behavior found in our simulations where the presence of stochasticity in the updating process is necessary for generating irregular IBIs.

Our work, however, does not rule out the possibility that the bursting behavior in brain slices is a manifestation of deterministic chaos. The demonstration that our stochastic model reproduces the experimentally observed behavior does not imply that other, possibly deterministic, models would not be equally (or more) successful in describing the experimental results. Our systematic analysis of the stochastic IBI data obtained from simulations of our network model points out several signatures of stochastic dynamics in the control behavior and in the results of surrogate analysis for the statistical significance of UPOs. These signatures have been discussed in detail in Secs. III and IV. It would be interesting to look for these signatures in brain-slice experiments. Such investigations would be very helpful in establishing the true nature of the collective dynamics of brain slices.

In our simulations, applications of external stimuli during control procedures do not alter the parameters (such as synaptic strengths, the relative strength of inhibition and time delays) that govern the network dynamics. This may not be true in networks of biological neurons. There are reasons to believe that repeated external stimulation causes permanent changes in the connectivity pattern of neuronal networks through synaptic plasticity. The phenomenon of kindling [22], for which the model studied here was originally developed [20], is a well-known example of changes in network properties caused by repeated external stimulation. Frequent stimulations may also alter the dynamics of biological networks by making the neurons fatigued or refractory. Evidence for stimulation-induced changes in network properties may be found in Ref. [18]. Any interpretation of the results of brain-slice experiments must take into account the possibility of such changes. These changes may also account for some of the differences between the results of our network 
simulation and those of brain-slice experiments.

Results of our surrogate analysis of IBI datasets for determining the statistical significance of UPO-like trajectories found in these purely stochastic time series cast serious doubts on the reliability of the statistical methods used in Refs. [15,16,19], especially for datasets of relatively small length. Our work suggests that far more stringent criteria and analysis are required for unambiguously establishing signatures of deterministic chaos in experimental time-series data.

\section{ACKNOWLEDGMENTS}

B.B. thanks S. J. Schiff for useful comments and for suggesting the demand pacing analysis, and D. Kaplan for useful discussions.
[1] H. Kantz and T. Schreiber, Nonlinear Time Series Analysis (Cambridge University Press, Cambridge, England, 1997).

[2] Nonlinear Dynamics and Brain Functions, edited by P.E. Rapp, N. Pradhan, and R. Sreenivasan (Nova Science, New York, 2000).

[3] D. Kaplan and L. Glass, Understanding Nonlinear Dynamics (Springer Verlag, Berlin, 1998).

[4] J. Fell, J. Roeschke, and P. Beckmann, Biol. Cybern. 69, 139 (1993).

[5] A. Babloyantz and A. Destexhe, Proc. Natl. Acad. Sci. U.S.A. 83, 3513 (1986).

[6] C.A. Skarda and W.J. Freeman, Behav. Brain Sci. 10, 161 (1987).

[7] I. Tsuda, Neural Networks 5, 313 (1992).

[8] L. Glass, in Handbook of Brain Theory and Neural Networks, edited by M.A. Arbib (MIT Press, Cambridge, MA, 1995), pp. 186-189.

[9] S.J. Schiff, K. Jerger, D.H. Duong, T. Chang, M.L. Spano, and W.L. Ditto, Nature (London) 370, 615 (1994).

[10] E. Ott, C. Grebogi, and J.A. Yorke, Phys. Rev. Lett. 64, 1196 (1990)

[11] J. Glanz, Science 265, 1174 (1994); 277, 1758 (1997).

[12] D.J. Christini and J.J. Collins, Phys. Rev. Lett. 75, 2782 (1995).

[13] D. Pierson and F. Moss, Phys. Rev. Lett. 75, 2124 (1995).

[14] P. So, E. Ott, S.J. Schiff, D.T. Kaplan, T. Sauer, and C. Grebogi, Phys. Rev. Lett. 76, 4705 (1996).

[15] P. So, E. Ott, T. Sauer, B.J. Gluckman, C. Grebogi, and S.J. Schiff, Phys. Rev. E 55, 5398 (1997).

[16] P. So, J.T. Francis, T.I. Netoff, B.J. Gluckman, and S.J. Schiff, Biophys. J. 74, 2776 (1998).

[17] M.W. Slutzky, P. Cvitanovic, and D.J. Mogul, Ann. Biomed. Eng. 29, 607 (2001).

[18] M.W. Slutzky, P. Cvitanovic, and D.J. Mogul (unpublished).

[19] M.L. Van Quyen, J. Martinerie, C. Adam, and F.J. Varela, Phys. Rev. E 56, 3401 (1997).

[20] M.R. Mehta, C. Dasgupta, and G.R. Ullal, Biol. Cybern. 68,
335 (1993); in Neural Modeling of Brain and Cognitive Disorders, edited by J.A. Reggia, E. Rupin, and R.S. Berndt (World Scientific, Singapore, 1996).

[21] B. Biswal and C. Dasgupta, Phys. Rev. Lett. 88, 088102 (2002).

[22] G.V. Goddard, D.C. McIntyre, and C.K. Leech, Exp. Neurol. 25, 295 (1969).

[23] D.J. Amit, Modeling Brain Function: The World of Attractor Neural Networks (Cambridge University Press, Cambridge, England, 1989).

[24] R.D. Traub, R. Miles, and J.R. Jefferys, J. Physiol. (London) 461, 525 (1993).

[25] S.J. Schiff, K. Jerger, T. Chang, T. Sauer, and P.G. Aitken, Biophys. J. 67, 684 (1994).

[26] Methods in Neuronal Modeling: From Ions to Networks, edited by C. Koch and I. Segev (MIT Press, Cambridge, MA, 1998).

[27] A. Garfinkel, M.L. Spano, W.L. Ditto, and J.N. Weiss, Science 257, 1230 (1992).

[28] D.J. Christini and J.J. Collins, Chaos 7, 544 (1997).

[29] D.J. Christini and D.T. Kaplan, Phys. Rev. E 61, 5149 (2000).

[30] W. Yang, M. Ding, A.J. Mandell, and E. Ott, Phys. Rev. E 51, 102 (1995)

[31] V. In, S.E. Mahan, W.L. Ditto, and M.L. Spano, Phys. Rev. Lett. 74, 4420 (1995).

[32] V. In, M.L. Spano, and M. Ding, Phys. Rev. Lett. 80, 700 (1998).

[33] R. Ramaswamy, S. Sinha, and N. Gupte, Phys. Rev. E 57, R2507 (1998).

[34] X. Pei and F. Moss, Nature (London) 379, 618 (1996).

[35] L. Menendez de la Prida, N. Stollenwerk, and J.V. SanchezAndres, Physica D 110, 323 (1997).

[36] J. Theiler, S. Eubank, A. Longtin, B. Galdrikian, and J.D. Farmer, Physica D 58, 77 (1992).

[37] B. Biswal, C. Dasgupta, and G. R. Ullal, Nonlinear Dynamics and Brain Functions (Ref. [2]).

[38] R.D. Traub and R. Dingledine, J. Neurophysiol. 64, 1009 (1990). 